\title{
INJECTIVE STRUCTURES
}

\author{
BY \\ J.-M. MARANDA
}

1. Introduction. In a category $\mathscr{C}$, an object $Q$ will be said to be injective with respect to a morphism $f: A \rightarrow B$ if for every $\phi: A \rightarrow Q$ there exists $\psi: B \rightarrow Q$ such that $\phi=\psi f$. An injective structure $\mathfrak{B}$ of $\mathscr{C}$ consists of a class $\mathfrak{S}$ of morphisms of $\mathscr{C}$, called the proper morphisms of $\mathfrak{B}$, and of a class $\mathfrak{Q}$ of objects of $\mathscr{C}$, called the injectives of $\mathfrak{B}$, such that (1) $\mathfrak{Q}$ is the class of all objects that are injective with respect to the morphisms in $\mathfrak{S},(2) \mathfrak{S}$ is the class of all morphisms with respect to which the objects in $\mathfrak{Q}$ are injective and (3) for any object $A$ in $\mathscr{C}$, there exists $\phi: A \rightarrow Q$ in $\mathfrak{S}$, where $Q \in \mathfrak{Q}$. An injective structure $\mathfrak{B}(\mathfrak{S}, \mathfrak{Q})$ of $\mathscr{C}$ will be called regular if for any objects $A$ in $\mathscr{C}$, the morphism $\phi: A \rightarrow Q$ in $\mathfrak{S}$, where $Q \in \mathbb{Q}$, may be chosen that if $f: A \rightarrow Q^{\prime}, Q^{\prime} \in \mathfrak{Q}$, then there is a unique $\psi: Q \rightarrow Q^{\prime}$ such that $f=\psi \phi$. ( $Q$ may then be called a $\mathfrak{B}$-completion of $A$.) One could consider an injective structure as a one-sided abelian structure with enough injectives in the sense of Heller [7]. All questions of relative homological algebra related to this notion of injective structure will be left for a later paper.

The author's aim in studying these structures on a category was to set up an abstract theory of, let us say, completions of modules, which would generalize the notions of rational completions of modules and of topological completions of modules defined by a set of ideals of the base ring, and the examples of injective structures that we will study will be related to these two processes of completion. There are, of course, other examples of injective structures on a category of modules, or its dual, or on other categories for that matter, but the ones we have chosen will suffice to illustrate the principles we wish to set forth.

We now describe the different sections of this work. $\$ 2$ is devoted to an elementary study of the notion of injective structure and related concepts. In $\$ 3$, we are concerned with the transfer of injective structures from one category to another through a functor with adjoint system. The notion of adjoint system generalizes that of an adjoint functor [9].

In $\S \S 4,5,6$ and 7 , we work in the category $\mathscr{C}_{R}$ of all (unitary) right modules over some ring $R$ (with unity element).

In $\S 4$, we give a natural definition of radicals in a category $\mathscr{C}_{R}$ and show that any radical is always obtained from an injective structure $\mathfrak{B}(\mathfrak{S}, \mathfrak{Q})$ by assigning to each module $A$ the kernel of any map $f: A \rightarrow Q$ in $\mathfrak{S}$, where $Q \in \mathfrak{Q}$. We also show that given any injective structure $\mathfrak{B}$, there is a coarsest regular injective structure $\mathfrak{B}^{*}$ finer than $\mathfrak{B}, \mathfrak{B}^{*}$ defining the same radical as $\mathfrak{B}$.

Received by the editors August 16, 1962. 
In $\S 5$, we show that given a regular injective structure $\mathfrak{B}(\circlearrowleft, \mathfrak{Q})$ of $\mathscr{C}_{R}$, it is possible to define a ring operation on the $\mathfrak{B}$-completion $\bar{R}$ of $R$ such that the canonical map from $R$ to $R$ is a ring homomorphism, and then every module in $\mathfrak{Q}$ may be turned into a right $\bar{R}$-module in a natural way. The section ends with a definition of a canonical correspondence between the $R$-submodules of an $R$-module $M$ and the $\bar{R}$-submodules of its $\mathfrak{B}$-completion $\bar{M}$.

In $\S 6$, we start by studying a notion of torsion radical of $\mathscr{C}_{R}$, which generalizes the ordinary notion of torsion subgroup of an abelian group. Torsion radicals are completely determined by certain sets of right ideals of $R$. Each torsion radical $\mathfrak{r}$ of $\mathscr{C}_{R}$ gives rise to two injective structures $\widetilde{\mathfrak{B}}_{\mathfrak{r}}$, which generalizes the notion of exact injective structure, and $\mathfrak{B}_{\mathfrak{r}}$, which is regular and which we call the raticnal injective structure determined by $\mathfrak{r}$. This work relies heavily on methods of $\operatorname{Baer}[1]$. The completion $\bar{R}$ of $R$ by $\mathfrak{B}_{\mathfrak{r}}$, turned into a ring, generalizes both the notion of ring of quotients studied in [8], [11] and [5], and the notion of ring of quotients of a commutative ring determined by any multiplicatively closed set of elements; we will call $R$ the right ring of quotients determined by $r$. The remainder of this section is devoted to the study of rings of quotients of $R$ determined by multiplicatively closed sets of ideals of $R$ each of which is invertible in some extension of $R$. It turns out that the left and right rings of quotients determined by such a set of ideals are equivalent ring extensions of $R$.

$\$ 7$ is a study of a generalization of the notion of pure subgroup of an abelian group. Given a set of ideals $\mathscr{E}$ of $R$, if $A$ is an $R$-submodule of $B$ in $\mathscr{C}_{R}$, then $A$ is said to be $\mathscr{E}$-pure in $B$ if $B N \cap A=A N$ for all $N \in \mathscr{E}$, while a homomorphism $f: A \rightarrow B$ is said to be $\mathscr{E}$-pure if $\operatorname{ker} f \subseteq \bigcap_{N \in \mathscr{E}} A N=\mathfrak{r}_{\mathscr{E}}(A)$ and if $f(A)$ is $\mathscr{E}$-pure in $B$. The class of all $\mathscr{E}$-pure homomorphisms is the class of proper maps of an injective structure $\mathfrak{B}_{\mathscr{E}}$ of $\mathscr{C}_{R}$.

If one assumes that $\mathscr{E}$ has the property " $N_{1}, N_{2} \in \mathscr{E}$ implies that there exists $N \in \mathscr{E}$ such that $N \subset N_{1} \cap N_{2}$," then for any module $A$ in $\mathscr{C}_{R},\{A N\}_{N \in \mathscr{E}}$ is a basis of the filter of neighborhoods of $O$ of a topology $\mathscr{T}(A, \mathscr{E})$ compatible with addition and scalar multiplication, the closure of $O$ in $A$ being $\mathfrak{r}_{\delta}(A)$. Then if $\kappa_{A}$ denotes the product of the natural homomorphism of $A$ onto $A / \mathfrak{r}_{\delta}(A)=A^{\prime}$ by the natural injection of $\left(A^{\prime}, \mathscr{T}\left(A^{\prime}, \mathscr{E}\right)\right)$ into its completion $S_{\mathscr{E}}(A)$, then there is an injective structure $\mathfrak{B}_{\delta}^{*}\left(\mathfrak{S}_{\delta}^{*}, \mathfrak{Q}_{\delta}^{*}\right)$ of $\mathscr{C}_{R}$ such that, for each module $A, \kappa_{A} \in \mathfrak{S}_{\delta}^{*}$ and $S_{\delta}(A) \in \mathbb{Q}_{\delta}^{*}$. This injective structure $\mathfrak{V}_{\delta}^{*}$ is finer than $\mathfrak{V}_{\delta}$ and coarser than $\mathfrak{B}_{\mathscr{\delta}}^{*}$. In general it is distinct from $\mathfrak{B}_{\mathscr{\delta}}$ and probably distinct from $\mathfrak{B}_{\mathscr{\&}}^{*}$. It was shown in [10] that when $\mathscr{E}$ is a multiplicatively closed set of non-null ideals of a principal ideal domain, then $\mathfrak{B}_{\&}=\mathfrak{B}_{\&}^{*}$.

2. Definition of and elementary results concerning injective structures. In any category $\mathscr{C}$ that we will consider it will be understood that the class of all morphisms from an object $A$ to an object $B$ is a set which we denote by $M(A, B)$. By the direct product of a family $\left\{A_{i}\right\}_{i \in I}$ of objects of $\mathscr{C}$, where $I$ is a set, we mean 
a family $\left\{\alpha_{i}: A \rightarrow A_{i}\right\}_{i \in I}$ such that, given any family $\left\{\beta_{i}: B \rightarrow A_{i}\right\}_{i \in I}$, there exists a unique $\phi: B \rightarrow A$ such that $\beta_{i}=\alpha_{i} \phi$ for all $i \in I$. (We will also say that $A$ is a direct product of the $A_{i}$.) Given two morphisms $f: A \rightarrow B$ and $g: A \rightarrow C$, we will say that the triple $(D, \beta, \gamma)$, where $\beta: B \rightarrow D$ and $\gamma: C \rightarrow D$ is a direct sum of $B$ and $C$ with $f$ and $g$ amalgamated if $\beta f=\gamma g$ and if given any triple $(X, \phi, \psi)$, where $\phi: B \rightarrow X$ and $\psi: C \rightarrow X$, if $\phi f=\psi g$, then there exists a unique $\chi: D \rightarrow X$ such that $\phi=\chi \beta$ and $\psi=\chi \gamma$. Given a morphism $f: A \rightarrow B$ and an object $C$ in the category $\mathscr{C}$, we will say that $C$ is injective with respect to $f$ if for any $\phi: A \rightarrow C$ there exists $\psi: B \rightarrow C$ such that $\phi=\psi f$. If $\mathbb{S}$ is a class of morphisms of $\mathscr{C}$, we will denote by $\Phi(\Im)$ the class of all objects of $\mathscr{C}$ that are injective with respect to all morphisms in $\mathfrak{S}$. This class $\Phi(\mathfrak{S})$ has the following properties:

A1. If $f: A \rightarrow B$ is a retraction and if $B \in \Phi(\Im)$, then $A \in \Phi(\Im)$.

A2. If $\left\{\phi_{i}: A \rightarrow A_{i}\right\}_{i \in I}$ is a direct product of $\left\{A_{i}\right\}_{i \in J}$, then $A \in \Phi(\mathcal{S})$ if and only if all the $A_{t} \in \Phi(\mathfrak{S})$.

If $\mathfrak{Q}$ is a class of objects of $\mathscr{C}$, we will denote by $\Psi(\mathfrak{Q})$ the class of all morphisms in $\mathscr{C}$ with respect to which the objects in $\mathbb{Q}$ are injective. This class $\Psi(\mathbb{Q})$ has the following properties:

B1. Any retraction is in $\Psi(Q)$.

B2. The product of two morphisms in $\Psi(\mathfrak{S})$ is also in $\Psi(\mathfrak{S})$.

B3. If $g f \in \Psi(\mathbb{Q})$, then $f \in \Psi(\mathbb{Q})$, and if $f$ is an epimorphism, then $g \in \Psi(\mathfrak{Q})$.

B4. If $f: A \rightarrow B$ is in $\Psi(\mathfrak{Q})$ and if $A \in \mathfrak{Q}$, then $f$ is a retraction.

B5. Given two morphisms $f: A \rightarrow B$ and $g: A \rightarrow C$, if $(D, \beta, \gamma)$ is a direct sum of $B$ and $C$ with $f$ and $g$ amalgamated and if $f \in \Psi(\mathbb{Q})$, then $\gamma \in \Psi(\mathbb{Q})$.

Finally, the functions $\Phi$ and $\Psi$ have the following properties:

C1. $\mathfrak{S} \subseteq \mathfrak{S}^{\prime}$ implies that $\Phi(\mathfrak{S}) \supseteq \Phi\left(\mathfrak{S}^{\prime}\right)$ and $\mathbb{Q} \subseteq \mathbb{Q}^{\prime}$ implies that $\Psi(\mathbb{Q}) \supseteq \Psi\left(\mathbb{Q}^{\prime}\right)$.

C2. Given families $\left\{\mathfrak{S}_{i}\right\}_{i \in I}$ and $\left\{\mathfrak{Q}_{i}\right\}_{i \in I}$, we have

$$
\begin{array}{ll}
\Phi\left(\bigcup_{i \in I} \mathfrak{S}_{i}\right)=\bigcap_{i \in I} \Phi\left(\mathfrak{S}_{i}\right), & \Phi\left(\bigcap_{i \in I} \mathfrak{S}_{i}\right) \supseteq \bigcup_{i \in I} \Phi\left(\mathfrak{S}_{i}\right), \\
\Psi\left(\bigcup_{i \in I} \mathfrak{Q}_{i}\right)=\bigcap_{i \in I} \Psi\left(\mathfrak{Q}_{i}\right), & \Psi\left(\bigcap_{i \in I} \mathbb{Q}_{i}\right) \supseteq \bigcup_{i \in I} \Psi\left(\mathfrak{Q}_{i}\right) .
\end{array}
$$

C3. For any $\mathfrak{S}$, there is a largest $\Im^{\prime}$ for which $\Phi\left(\Im^{\prime}\right)=\Phi(\Im)$, namely $\mathfrak{S}^{\prime}=\Psi \Phi(\mathfrak{S})$, and for any $\mathbb{Q}$, there is a largest $\mathfrak{Q}^{\prime}$ for which $\Psi\left(\mathbb{Q}^{\prime}\right)=\Psi(\mathbb{Q})$, namely $\mathfrak{Q}^{\prime}=\Phi \Psi(\mathfrak{Q})$.

Definition 1. A couple $(\mathfrak{S}, \mathfrak{Q})$, where $\mathfrak{S}$ is a class of morphisms and $\mathfrak{Q}$ is a class of objects of $\mathscr{C}$, will be said to define an injective structure $\mathfrak{B}=\mathfrak{B}(\mathfrak{S}, \mathfrak{Q})$ of $\mathscr{C}$ if

D1. $\Phi(\Im)=\mathfrak{Q}$.

D2. $\Psi(\mathfrak{Q})=\mathfrak{G}$.

D3. For any object $A$ of $\mathscr{C}$, there exists a morphism $f: A \rightarrow Q$ in $\mathfrak{S}$, where $Q \in \mathbb{Q}$.

We will call $\subseteq$ and $\mathfrak{Q}$ the class of proper morphisms and the class of injectives of $\mathfrak{B}$, respectively. 
Proposition 1. If $\mathfrak{B}(\mathfrak{G}, \mathbb{Q})$ is an injective structure of $\mathscr{C}$, then an object $A$ of $\mathscr{C}$ is in $\mathfrak{Q}$ if and only if every morphism $f: A \rightarrow B$ in $\mathfrak{S}$ is a retraction.

Proof. The necessity is immediate by B4. Assume that every morphism $f: A \rightarrow B$ in $\mathfrak{S}$ is a retraction. By D3, $f$ may be chosen such that $B \in \mathbb{Q}=\Phi(\Im)$. Then, by $\mathrm{A} 2, A \in \mathbb{Q}$.

Definition 2. Given an injective structure $\mathfrak{B}(\mathfrak{S}, \mathfrak{Q})$ of $\mathscr{C}$, a subclass $\mathfrak{Q}^{\prime}$ of $\mathfrak{Q}$ will be called

(a) a base of injectives of $\mathfrak{B}$ if every object in $\mathfrak{Q}$ is a retract of an object in $\mathbb{Q}^{\prime}$,

(b) a subbase of injectives of $\mathfrak{B}$ if the class of all direct products of objects in $\mathfrak{Q}^{\prime}$ is a base of injectives of $\mathfrak{B}$,

(c) a generating class of injectives of $\mathfrak{B}$ if $\Psi\left(\mathfrak{Q}^{\prime}\right)=\mathfrak{S}$.

It is obvious that a base of injectives of $\mathfrak{B}$ is a subbase of injectives of $\mathfrak{B}$.

LEMMA 1. If $\mathfrak{Q}^{\prime \prime}, \mathbb{Q}^{\prime}$ and $\mathfrak{Q}$ are classes of objects of $\mathscr{C}$, if every object in $\mathbb{Q}^{\prime}$ is a direct product of objects in $\mathfrak{Q}^{\prime \prime}$, and if every object in $\mathfrak{Q}$ is a retract of an object in $\mathfrak{Q}^{\prime}$, then $\Psi\left(\mathfrak{Q}^{\prime \prime}\right) \subseteq \Psi\left(\mathfrak{Q}^{\prime}\right) \subseteq \Psi(\mathfrak{Q})$.

Proof. Since $\mathfrak{Q}^{\prime \prime} \subseteq \Phi \Psi\left(Q^{\prime \prime}\right)$, by $A 2, \mathfrak{Q}^{\prime} \subseteq \Phi \Psi\left(Q^{\prime \prime}\right)$ so that $\Psi\left(Q^{\prime}\right) \supseteq \Psi \Phi \Psi\left(Q^{\prime \prime}\right)$ $=\Psi\left(Q^{\prime \prime}\right)$ by $C 1$ and $C 3$. Since $Q^{\prime} \subseteq \Phi \Psi\left(Q^{\prime}\right)$, by $A 1, \mathbb{Q} \subseteq \Phi \Psi\left(Q^{\prime}\right)$ so that $\Psi(\mathfrak{Q}) \supseteq \Psi \Phi \Psi\left(\mathfrak{Q}^{\prime}\right)=\Psi\left(\mathfrak{Q}^{\prime}\right)$ by $\mathrm{C} 1$ and $\mathrm{C} 3$.

CoRollary. If $\mathfrak{B}(\mathfrak{S}, \mathbb{Q})$ is an injective structure of $\mathscr{C}$ then any subbase $\mathfrak{Q}^{\prime}$ of injectives of $\mathfrak{B}$ is a generating class of injectives of $\mathfrak{B}$.

Proof. By Lemma $1, \Psi\left(\mathfrak{Q}^{\prime}\right) \subseteq \Psi(\mathfrak{Q})$. However, since $\mathfrak{Q}^{\prime} \subseteq \mathfrak{Q}, \Psi\left(\mathfrak{Q}^{\prime}\right) \supseteq \Psi(\mathfrak{Q})$.

Proposition 2. A class $\mathfrak{Q}^{\prime}$ of objects of $\mathscr{C}$ is a base of injectives of an injective structure of $\mathscr{C}$ if and only if given any object $A$ of $\mathscr{C}$, there exists $\phi: A \rightarrow Q$ in $\Psi\left(\mathfrak{Q}^{\prime}\right)$, where $Q \in \mathbb{Q}^{\prime}$.

Proof. The necessity of this condition is obvious by B1 and B2. Assume the condition holds, let $\mathbb{Q}$ be the class of all retracts of objects in $\mathbb{Q}^{\prime}$, and let $\subseteq=\Psi\left(Q^{\prime}\right)$. Obviously, $\mathfrak{Q}^{\prime} \subseteq \mathbb{Q}$ so that $\Psi\left(\mathbb{Q}^{\prime}\right) \supseteq \Psi(\mathfrak{Q})$ and then by Lemma $1, \Psi\left(\mathfrak{Q}^{\prime}\right)=\Psi(\mathfrak{Q})$. The proposition will then obviously be proved as soon as we can show that $\Phi(\mathfrak{S})=\mathfrak{Q}$. Evidently, $\mathfrak{Q} \subseteq \Phi \Psi(\mathbb{Q})=\Phi(\mathfrak{S})$. Let $A \in \Phi(\mathfrak{S})$. There exists $\phi: A \rightarrow Q$ in $\Psi\left(Q^{\prime}\right)=\mathfrak{S}$, where $Q \in \mathbb{Q}^{\prime}$, so that there exists $\psi: Q \rightarrow A$ such that $\psi \phi=1_{A}$, i.e., $\phi$ is a retraction and therefore $A \in \mathfrak{Q}$.

Proposition 3. If $\mathscr{C}$ has direct products then any set $\mathbb{Q}^{\prime \prime}$ of objects of $\mathscr{C}$ is a subbase of injectives of an injective structure of $\mathscr{C}$.

Proof. Let $\mathfrak{Q}^{\prime}$ be the class of all objects that are direct products of objects in $\mathfrak{Q}^{\prime \prime}$. If $A$ is any object of $\mathscr{C}$, let $\left\{\phi_{i}: A \rightarrow Q_{i}\right\}_{i \in I}$ be the set of all morphisms from $A$ to objects in $Q^{\prime \prime}$ and let $\left(Q,\left\{\alpha_{i}\right\}_{i \in I}\right)$ be a direct product of $\left\{Q_{i}\right\}_{i \in I}$. There exists $\phi: A \rightarrow Q$ such that $\phi_{i}=\alpha_{i} \phi$ for all $i \in I$. That $\phi \in \Psi\left(Q^{\prime \prime}\right)$ is obvious since if 
$f: A \rightarrow Q^{\prime \prime} \in Q^{\prime \prime}$, then there exists $i \in I$ such that $f=\phi_{i}$ and $Q^{\prime \prime}=Q_{i}$ so that $f=\alpha_{i} \phi$. But by Lemma $1, \Psi\left(Q^{\prime \prime}\right) \subseteq \Psi\left(\mathfrak{Q}^{\prime}\right)$ so that by Proposition $2, \mathfrak{Q}^{\prime}$ is a base of injectives of an injective structure $\mathfrak{B}(\mathfrak{S}, \mathfrak{Q})$ of $\mathscr{C}$ and $\mathbb{Q}^{\prime \prime}$, is of course, a subbasis of injectives of $\mathfrak{B}$.

DEFINITION 3. An injective structure $\mathfrak{B}(\mathfrak{S}, \mathfrak{Q})$ of $\mathscr{C}$ will be called regular if for every object $A$ of $\mathscr{C}$, there exists a morphism $f: A \rightarrow Q$ in $\mathfrak{S}$, where $Q \in \mathbb{Q}$, such that for any $\phi: A \rightarrow Q^{\prime} \in \mathbb{Q}$, there exists a unique $\psi: Q \rightarrow Q^{\prime}$ such that $\phi=\psi f$.

Proposition 4. A class $\mathfrak{Q}$ of objects of $\mathscr{C}$ is the class of injectives of a regular injective structure of $\mathscr{C}$ if and oniy if for any objects $A$ of $\mathscr{C}$, there exists $f_{A}: A \rightarrow Q_{A}$, where $Q_{A} \in \mathbb{Q}$, such that for any $\phi: A \rightarrow Q^{\prime}$, where $Q^{\prime} \in \mathbb{Q}$, there exists a unique $\psi: Q_{A} \rightarrow Q^{\prime}$ such that $\phi=\psi f_{A}$.

Proof. The necessity of this condition is immediate. Assume the condition holds. Then, by Proposition 2, $\mathfrak{Q}$ is a basis of injectives of an injective structure of $\mathscr{C}$. If $A$ is an injective of this structure, then there exists $\phi: Q_{A} \rightarrow A$ such that $1_{A}=\phi f_{A}$. Then, $f_{A} \phi f_{A}=f_{A}=1_{Q_{A}} f_{A}$ so that $f_{A} \phi=1_{Q_{A}}$. This means that $f_{A}$ is an isomorphism.

Definition 4. If $\mathfrak{B}(\mathfrak{S}, \mathfrak{Q})$ and $\mathfrak{B}^{\prime}\left(\mathfrak{S}^{\prime}, \mathfrak{Q}^{\prime}\right)$ are two injective structures of $\mathscr{C}$, then we will say that $\mathfrak{B}$ is coarser than $\mathfrak{B}^{\prime}$ or that $\mathfrak{B}^{\prime}$ is finer than $\mathfrak{B}$ if $\mathfrak{S} \supseteq \mathbb{S}^{\prime}$, or equivalently, if $\mathbb{Q} \subseteq \mathbb{Q}^{\prime}$.

It is obvious that the injective structures of $\mathscr{C}$ are partially ordered by this relation. Also, there is a finest injective structure on $\mathscr{C}$ whose proper morphisms are just the retractions and whose class of injectives is the class of all objects of $\mathscr{C}$. If $\mathscr{C}$ is a category of all modules and homomorphisms over some ring, then the class of all homomorphisms is the class of proper morphisms of the coarsest injective structure of $\mathscr{C}$, the class of injectives of this structure being just the class of all 0 -modults.

Proposition 5. If $\mathscr{C}$ is a category with direct products and if $\left\{\mathfrak{B}_{i}\left(\mathfrak{S}_{i}, \mathbb{Q}_{i}\right)\right\}_{i \in I}$ is a family of injective structures of $\mathscr{C}$, where $I$ is a set, then this family has a least upper bound $\mathfrak{B}(\mathfrak{S}, \mathfrak{Q})=\sup _{i \in I} \mathfrak{B}_{i}$, where $\mathfrak{S}=\bigcap_{i \in I} \mathbb{E}_{i}$. Furthermore, if for each $i \in I, \mathfrak{Q}_{i}^{\prime \prime}$ is a subbase of injectives of $\mathfrak{B}_{i}$, then $\bigcup_{i \in I} \mathfrak{Q}_{i}^{\prime \prime}$ is a subbase of injectives of $\mathfrak{B}$.

Proof. For each $i \in I$, let $\mathfrak{Q}_{i}^{\prime}$ be the class of all direct products of objects in $\mathbb{Q}_{i}^{\prime \prime}$ Then, by the associativity of direct products, the class $Q^{\prime}$ of all direct products of objects in $\bigcup_{i \in I} \mathfrak{Q}_{i}^{\prime}$ is also the class of all direct products of objects in $\bigcup_{i \in I} \mathfrak{Q}_{i}^{\prime \prime}$. Let $A$ be any object of $\mathscr{C}$. For each $i \in I$, there exists $\phi_{i}: A \rightarrow Q_{i}$ in $\mathfrak{S}_{i}$, where $Q_{i} \in \mathbb{Q}_{i}^{\prime}$. Let $\left(Q,\left\{\alpha_{i}\right\}_{i \in I}\right)$ be a direct product of $\left\{Q_{i}\right\}_{i \in I}$. There exists $\phi: A \rightarrow Q$ such that $\phi_{i}=\alpha_{i} \phi$ for each $i \in I$. If $f: A \rightarrow Q_{i}^{\prime} \in \mathbb{Q}_{i}^{\prime}$, there exists $g: Q_{i} \rightarrow Q_{i}^{\prime}$ such that $f=g \phi_{i}$ so that $f=\left(g \alpha_{i}\right) \phi$. Then, we have $Q \in \mathbb{Q}^{\prime}$ and $\phi \in \Psi\left(\bigcup_{i \in I} \mathfrak{Q}_{i}^{\prime}\right) \subseteq \Psi\left(\mathbb{Q}^{\prime}\right)$ by Lemma 1 so that, by Proposition 2, $Q^{\prime}$ is a base of injectives, and therefore 
$\bigcup_{i \in I} \mathfrak{Q}^{n}{ }_{i}$ is a subbase of injectives of an injective structure $\mathfrak{B}(\mathfrak{S}, \mathfrak{Q})$ of $\mathscr{C}$. By the corollary of Lemma 1, and by $\mathrm{C} 2$,

$$
\mathfrak{S}=\Psi\left(\bigcup_{i \in I} \mathfrak{Q}_{i}^{\prime \prime}\right)=\bigcap_{i \in I} \Psi\left(Q_{i}^{\prime \prime}\right)=\bigcap_{i \in I} \mathfrak{S}_{i}
$$

so that $\mathfrak{B}$ is obviously a least upper bound of $\left\{\mathfrak{V}_{i}\right\}_{i \in I}$.

Let us consider some examples. Throughout this paper, we will only consider rings with unity element. If $R$ is such a ring, $\mathscr{C}_{R}$ will denote the category whose objects are the right $R$-modules and whose morphisms are the $R$-homomorphisms. It is well known that the class of all ordinary injective modules of $\mathscr{C}_{R}$ is the class of injectives of an injective structure of $\mathscr{C}_{R}$, which we will call the exact injective structure of $\mathscr{C}_{R}$, whose class of proper homomorphisms is just the class of all monomorphisms.

Now let $R$ be a principal ideal domain and let $\mathscr{S}$ be a set of primes in $R$. If $Q^{\prime}$ is a complete set of representatives of the class of all $R$-modules of type $p^{n}$, where $p \in \mathscr{S}$ and $n$ is a natural number or $\infty$, or where $p \notin \mathscr{S}$ and $n$ is $\propto$, then, by Proposition $3, Q^{\prime}$ is a subbase of injectives of an injective structure $\mathfrak{B}_{\mathscr{S}}$ of $\mathscr{C}_{R}$, and it was shown in [11] that the proper homomorphisms of $\mathfrak{V}_{\mathscr{S}}$ are just the $\mathscr{S}$-pure monomorphisms. If one deletes from $\mathfrak{Q}^{\prime}$ all the modules of type $p^{\infty}$, then one has a subbasis of injectives of an injective structure $\mathfrak{B}_{\mathscr{S}}^{\prime}$ of $\mathscr{C}_{R}$ whose proper homomorphisms are just the $\mathscr{S}$-pure homomorphisms $[12, \S 4] ; \mathfrak{B}_{\mathscr{S}}$ is just the l.u.b. of $\mathfrak{B}_{\mathscr{S}}^{\prime}$ with the exact injective structure of $\mathscr{C}_{R}$. By Proposition $5, \mathfrak{B}_{\mathscr{S}}^{\prime}$ is the l.u.b. of all the $\mathfrak{B}_{p}^{\prime}, p$ running through the primes in $\mathscr{S}$. From results in [10], one may deduce easily that $\mathfrak{P}_{\mathscr{S}}^{\prime}$ is regular.

3. Adjoint systems and adjoint functors. In this section we generalize somewhat some of the results in [9]. For any category $\mathscr{C}$, we will denote the class of objects of $\mathscr{C}$ by $\overline{\mathscr{C}}$. If $T$ is a functor defined on $\mathscr{C}$, then we denote the restriction of $T$ to $\overline{\mathscr{C}}$ by $T$.

Definition 1. Given a functor $T: \mathscr{C}^{\prime} \rightarrow \mathscr{C}$, a left adjoint system of $T$ is a couple $(S, \kappa)$, where $S$ is a function from $\overline{\mathscr{C}}$ to $\overline{\mathscr{C}}^{\prime}$, where $\kappa$ is a family of morphisms $\left\{\kappa_{A}: A \rightarrow T S(A)\right\}_{A \in \overline{\mathscr{C}}}$ and where for each $A \in \overline{\mathscr{C}}$, if $f: A \rightarrow T\left(A^{\prime}\right)$, then there exists $g: S(A) \rightarrow A^{\prime}$ such that $f=T(g) \kappa_{A}$.

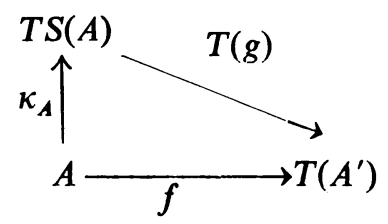

Proposition 1. Given a functor $T: \mathscr{C}^{\prime} \rightarrow \mathscr{C}$, if $T$ has a left adjoint system $(S, \kappa)$ and if for each $g: S(A) \rightarrow B^{\prime}$, one sets $\alpha_{A B^{\prime}}(g)=T(g) \kappa_{A}: A \rightarrow T\left(B^{\prime}\right)$ then $\alpha_{A B^{\prime}}$ is a mapping of $M\left(S(A), B^{\prime}\right)$ onto $M\left(A, T\left(B^{\prime}\right)\right), \alpha_{A}=\left\{\alpha_{A B^{\prime}}\right\}_{B^{\prime} \in \overline{\mathscr{C}^{\prime}}}$ is a natural transformation from $M\left(S(A), \mathscr{C}^{\prime}\right)$ to $M\left(A, T\left(\mathscr{C}^{\prime}\right)\right)$, and $\kappa_{A}=\alpha_{A, S(A)}\left(1_{S(A)}\right)$. 
Conversely, given a function $S: \overline{\mathscr{C}} \rightarrow \overline{\mathscr{C}}^{\prime}$, and given, for each $A \in \overline{\mathscr{C}}$, a natural transformation $\alpha_{A}: M\left(S(A), \mathscr{C}^{\prime}\right) \rightarrow M\left(A, T\left(\mathscr{C}^{\prime}\right)\right)$, where for each $B^{\prime} \in \overline{\mathscr{C}}^{\prime}, \alpha_{A B^{\prime}}$ is surjective, then if one sets $\kappa=\left\{\kappa_{A}=\alpha_{A, S(A)}\left(1_{S(A)}\right)\right\}_{A \in \overline{\mathscr{C}}},(S, \kappa)$ is a left adjoint system of $T$ defining the given $\alpha_{A}$ as above.

Proof. Assume first of all that $(S, \kappa)$ is a left adjoint system of $T$. Then it is obvious that each $\alpha_{A B^{\prime}}$ is surjective. Let us show that each $\alpha_{A}$ is a natural transformation. We must show that if $f^{\prime}: A^{\prime} \rightarrow B^{\prime}$ in $\mathscr{C}^{\prime}$, then the diagram

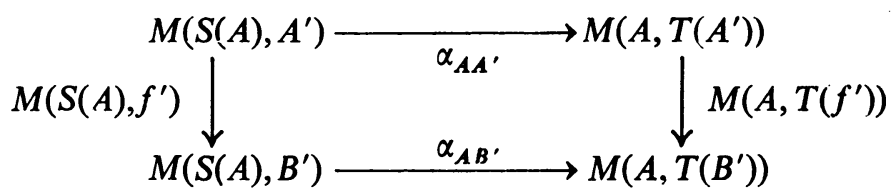

is commutative. But for each $g^{\prime} \in M\left(S(A), A^{\prime}\right)$,

$$
\begin{aligned}
M\left(A, T\left(f^{\prime}\right)\right) \alpha_{A A^{\prime}}\left(g^{\prime}\right) & =M\left(A, T\left(f^{\prime}\right)\right) T\left(g^{\prime}\right) \kappa_{A}=T\left(f^{\prime}\right) T\left(g^{\prime}\right) \kappa_{A} \\
& =T\left(f^{\prime} g^{\prime}\right) \kappa_{A}=\alpha_{A B^{\prime}}\left(f^{\prime} g^{\prime}\right)=\alpha_{A B^{\prime}} M\left(S(A), f^{\prime}\right)\left(g^{\prime}\right) .
\end{aligned}
$$

That each $\kappa_{A}=\alpha_{A S(A)}\left(1_{S(A)}\right)$ is obvious. Conversely, assume that there exists a function $S: \overline{\mathscr{C}}_{\rightarrow} \rightarrow \overline{\mathscr{C}}^{\prime}$ and, for each $A \in \overline{\mathscr{C}}$, a natural transformation $\alpha_{A}$ from $M\left(S(A), \mathscr{C}^{\prime}\right)$ to $M\left(A, T\left(\mathscr{C}^{\prime}\right)\right)$ where for each $B^{\prime} \in \overline{\mathscr{C}}^{\prime}, \alpha_{A B^{\prime}}$ is surjective. For each $A \in \overline{\mathscr{C}}$, set

$$
\kappa_{A}=\alpha_{A S(A)}\left(1_{S(A)}\right): A \rightarrow T S(A) .
$$

Then, if $f: A \rightarrow T\left(B^{\prime}\right)$, there exists $g^{\prime}: S(A) \rightarrow B^{\prime}$ such that $\alpha_{A B^{\prime}}\left(g^{\prime}\right)=f$, and from the commutativity of the diagram

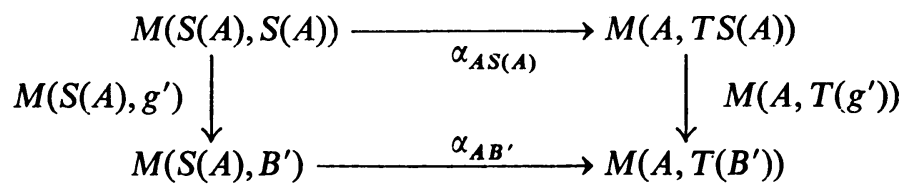

we deduce that

$$
\begin{aligned}
T\left(g^{\prime}\right) \kappa_{A} & =T\left(g^{\prime}\right) \alpha_{A S(A)}\left(1_{S(A)}\right)=M\left(A, T\left(g^{\prime}\right)\right) \alpha_{A S(A)}\left(1_{S(A)}\right) \\
& =\alpha_{A B^{\prime}} M\left(S(A), g^{\prime}\right)\left(1_{S(A)}\right)=\alpha_{A B^{\prime}}\left(g^{\prime} 1_{S(A)}\right)=\alpha_{A B^{\prime}}\left(g^{\prime}\right)=f .
\end{aligned}
$$

Proposition 2. Let $T: \mathscr{C}^{\prime} \rightarrow \mathscr{C}$ and $S: \mathscr{C} \rightarrow \mathscr{C}^{\prime}$ be functors, let $(S, \kappa)$ be a left adjoint system of $T$, and let the $\alpha_{A}$ be the natural transformations described in the preceding proposition. The following two statements are equivalent:

(1) $\kappa$ is a natural transformation from $1_{\mathscr{C}}$ to $T S$.

(2) $\alpha=\left\{\alpha_{A}\right\}_{A \in \bar{q}}$ is a natural transformation from $M\left(S(\mathscr{C}), \mathscr{C}^{\prime}\right)$ to $M\left(\mathscr{C}, T\left(\mathscr{C}^{\prime}\right)\right)$.

When these conditions are satisfied we say that $\kappa$ or $\alpha$ defines $S$ as a left adjoint functor of $T$. 
Proof. Assume first of all that $\kappa$ is a natural transformation, and let $f: A \rightarrow B$ in $\mathscr{C}$. We must show that the diagram

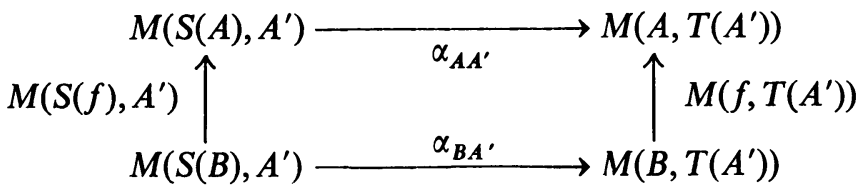

is commutative for any $A^{\prime} \in \overline{\mathscr{C}}^{\prime}$. But if $g^{\prime} \in M\left(S(B), A^{\prime}\right)$, we have

$$
\begin{aligned}
\alpha_{A A^{\prime}} M\left(S(f), A^{\prime}\right)\left(g^{\prime}\right) & =\alpha_{A A^{\prime}}\left(g^{\prime} S(f)\right)=T\left(g^{\prime} S(f)\right) \kappa_{A}=T\left(g^{\prime}\right)\left(T S(f) \kappa_{A}\right) \\
& =T\left(g^{\prime}\right) \kappa_{B} f=\alpha_{B A^{\prime}}\left(g^{\prime}\right) f=M\left(f, T\left(A^{\prime}\right)\right) \alpha_{B A^{\prime}}\left(g^{\prime}\right) .
\end{aligned}
$$

Conversely, assume that $\alpha$ is a natural transformation, and let $f: A \rightarrow B$ in $\mathscr{C}$. From the commutativity of the diagram

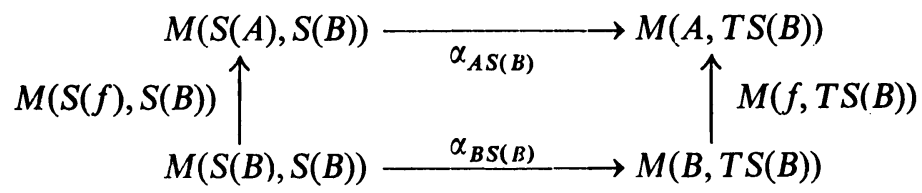

we deduce that

$$
\begin{aligned}
T S(f) \kappa_{A} & =\alpha_{A S(B)}(S(f))=\alpha_{A S(B)}\left(1_{S(B)} S(f)\right) \\
& =\alpha_{A S(B)} M(S(f), S(B))\left(1_{S(B)}\right)=M(f, T S(B)) \alpha_{B S(B)}\left(1_{S(B)}\right) \\
& =M(f, T S(B))\left(\kappa_{B}\right)=\kappa_{B} f
\end{aligned}
$$

so that $\kappa$ is a natural transformation.

Definition 2. Given a functor $T: \mathscr{C}^{\prime} \rightarrow \mathscr{S}$, a left adjoint system $(S, \kappa)$ of $T$ will be called regular if, given any $f: A \rightarrow T\left(A^{\prime}\right)$, there exists a unique $g: S(A) \rightarrow A^{\prime}$ such that $f=T(g) \kappa_{A}$, i.e., if for each $A \in \overline{\mathscr{C}}$ the natural transformations $\alpha_{A}$ defined in Theorem 1 are composed of biunique and surjective maps. If $S$ is a left adjoint of $T$ and if $\kappa: 1_{\varnothing} \rightarrow T S$ is a natural transformation, then we will say that $S$ is a regular left adjoint of $T$ defined by $\kappa$ if $(S, \kappa)$ is a regular left adjoint system of $T$.

Proposition 3. Given a functor $T: \mathscr{C}^{\prime} \rightarrow \mathscr{C}$ and a regular left adjoint system $(S, \kappa)$ of $T$, there exists a unique left adjoint of $T$, which we also denote by $S$, defined by $\kappa$.

Proof. If such a functor $S$ exists, then for every $f: A \rightarrow B$ in $\mathscr{C}, S(f)$ must be the only morphism from $S(A)$ to $S(B)$ for which the diagram

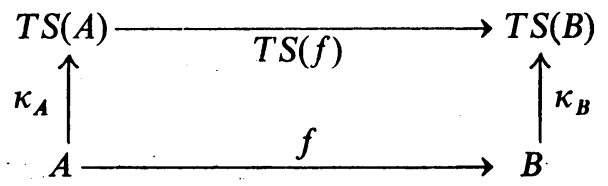


is commutative. Let us show that this effectively defines a functor. For every $A \in \overline{\mathscr{C}}$, since $\kappa_{A}=T\left(1_{S(A)}\right) \kappa_{A}, 1_{S(A)}=S\left(1_{A}\right)$. If $f: A \rightarrow B$ and $g: B \rightarrow C$, since $T S(f) \kappa_{A}=\kappa_{B} f$ and $T S(g) \kappa_{B}=\kappa_{C} g$,

$$
T(S(g) S(f)) \kappa_{A}=T S(g)(T S(f)) \kappa_{A}=\left(T S(g) \kappa_{B}\right) f=\kappa_{C}(g f)
$$

so that $S(g) S(f)=S(g f)$.

Proposition 4. Given a functor $T: \mathscr{C}^{\prime} \rightarrow \mathscr{C}$, any two regular left adjoints $S$ and $S^{\prime}$ of $T$ are equivalent.

Proof. Let $\kappa: 1_{\mathscr{\zeta}} \rightarrow T S$ and $\kappa^{\prime}: 1_{\mathscr{C}} \rightarrow T S^{\prime}$ be natural transformations defining $S$ and $S^{\prime}$ as regular left adjoints of $T$. For each $A \in \overline{\mathscr{C}}$, there exist unique morphisms $\zeta_{A}: S(A) \rightarrow S^{\prime}(A)$ and $\eta_{A}: S^{\prime}(A) \rightarrow S(A)$ such that $\kappa_{A}^{\prime}=T\left(\zeta_{A}\right) \kappa_{A}$ and $\kappa_{A}=T\left(\eta_{A}\right) \kappa_{A}^{\prime}$. Then

$$
\begin{aligned}
& \kappa_{A}^{\prime}=T\left(\zeta_{A}\right) T\left(\eta_{A}\right) \kappa_{A}^{\prime}=T\left(\zeta_{A} \eta_{A}\right) \kappa_{A}^{\prime}, \\
& \kappa_{A}=T\left(\eta_{A}\right) T\left(\zeta_{A}\right) \kappa_{A}=T\left(\eta_{A} \zeta_{A}\right) \kappa_{A}
\end{aligned}
$$

so that $\zeta_{A} \eta_{A}=1_{S^{\prime}(A)}$ and $\eta_{A} \zeta_{A}=1_{S(A)}$, i.e., $\zeta_{A}$ and $\eta_{A}$ are isomorphisms and inverses of one another. Now we show that $\zeta=\left\{\zeta_{A}\right\}_{A \in \overline{\mathcal{Y}}}$ is a natural transformation. Let $f: A \rightarrow B$ in $\mathscr{C}$. Since the two diagrams
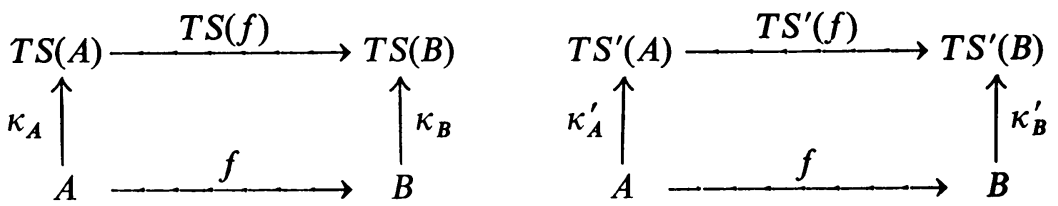

are commutative, we have

$$
\begin{aligned}
T\left(S^{\prime}(f) \zeta_{A}\right) \kappa_{A} & =T S^{\prime}(f)\left(T\left(\zeta_{A}\right) \kappa_{A}\right)=T S^{\prime}(f) \kappa_{A}^{\prime}=\kappa_{B}^{\prime} f=T\left(\zeta_{B}\right) \kappa_{B} f \\
& =T\left(\zeta_{B}\right) T S(f) \kappa_{A}=T\left(\zeta_{B} S(f)\right) \kappa_{A}
\end{aligned}
$$

so that $S^{\prime}(f) \zeta_{A}=\zeta_{B} S(f)$. Then, $\eta=\left\{\eta_{A}\right\}_{A \in \bar{G}}$ must also be a natural transformation.

Given two functors $T: \mathscr{C}^{\prime} \rightarrow \mathscr{C}$ and $T^{\prime}: \mathscr{C}^{\prime \prime} \rightarrow \mathscr{C}^{\prime}$. If $(S, \kappa)$ and $\left(S^{\prime}, \kappa^{\prime}\right)$ are left adjoint systems of $T$ and $T^{\prime}$, respectively, and if, for each $A \in \overline{\mathscr{C}}$, one sets $\kappa_{A}^{\prime \prime}=T\left(\kappa_{S(A)}^{\prime}\right) \kappa_{A}$, then $\left(S^{\prime} S, \kappa^{\prime \prime}\right)$ is a left adjoint system of $T T^{\prime}$ which we will call the product of the two given systems. If $A \in \overline{\mathscr{C}}$ and if $f: A \rightarrow T T^{\prime}\left(A^{\prime \prime}\right)$, there exists $g: S(A) \rightarrow T^{\prime}\left(A^{\prime \prime}\right)$ such that $f=T(g) \kappa_{A}$ and then there exists $h: S^{\prime} S(A) \rightarrow A^{\prime \prime}$ such that $g=T^{\prime}(h) \kappa_{S(A)}^{\prime}$ so that $f=T T^{\prime}(h)\left(T\left(\kappa_{S(A)}^{\prime}\right) \kappa_{A}\right)$.

If the $\alpha_{A}$ and the $\alpha_{A}^{\prime}$, are the natural transformations associated with $(S, \kappa)$ and $\left(S^{\prime}, \kappa^{\prime}\right)$, respectively, then it is easy to see that the $\alpha_{A} \alpha_{S_{(A)}^{\prime}}^{\prime}$ are the natural transformations associated with $\left(S^{\prime} S, \kappa^{\prime \prime}\right)$.

Now assume that the functors $S: \mathscr{C} \rightarrow \mathscr{C}^{\prime}$ and $S^{\prime}: \mathscr{C}^{\prime} \rightarrow \mathscr{C}^{\prime \prime}$ are left adjoints of $T$ and $T^{\prime}$ defined by the natural transformations $\kappa: 1_{\mathscr{Q}} \rightarrow T S$ and $\kappa^{\prime}: 1_{\mathscr{Q}^{\prime}} \rightarrow T^{\prime} S^{\prime}$, respectively, and let $\alpha: M\left(S(\mathscr{C}), \mathscr{C}^{\prime}\right) \rightarrow M\left(\mathscr{C}, T\left(\mathscr{C}^{\prime}\right)\right)$ and $\alpha^{\prime}: M\left(S^{\prime}\left(\mathscr{C}^{\prime}\right), \mathscr{C}^{\prime \prime}\right)$ 
$\rightarrow M\left(\mathscr{C}^{\prime}, T^{\prime}\left(\mathscr{C}^{\prime \prime}\right)\right)$ be the natural transformations corresponding to $\kappa$ and $\kappa^{\prime}$, respectively.

Then, using the notation of [6, Appendix], $S^{\prime} S$ is a left adjoint functor of $T T^{\prime}$ defined by the natural transformation $\left(T * \kappa^{\prime} * S\right) \kappa: 1_{\varnothing} \rightarrow T T^{\prime} S^{\prime} S$ to which is associated the natural transformation

$$
\alpha\left(\alpha^{\prime} * S\right): M\left(S^{\prime} S(\mathscr{C}), \mathscr{C}^{\prime \prime}\right) \rightarrow M\left(\mathscr{C}, T T^{\prime}\left(\mathscr{C}^{\prime \prime}\right)\right) .
$$

It is trivially true that the product of two regular left adjoint systems or of two regular adjoint functors is again regular.

We now notice that if $Q^{\prime}$ is a set of objects of $\mathscr{C}$ which is a basis of injectives of an injective structure $\mathfrak{B}(\mathfrak{S}, \mathfrak{Q})$ of $\mathscr{C}$, if $\mathscr{C}^{\prime}$ denotes the complete subcategory of $\mathscr{C}$ whose objects are the objects in $\mathfrak{Q}^{\prime}$, and if $T$ denotes the natural injection of $\mathscr{C}^{\prime}$ in $\mathscr{C}$, then $T$ has a left adjoint system $(S, \kappa)$ obtained by choosing for any object $A$ of $\mathscr{C}$ any map $\kappa_{A}: A \rightarrow S(A)$ in $\mathcal{S}$, where $S(A) \in \mathbb{Q}^{\prime}$. We will call $(S, \kappa)$ a description of $\mathfrak{B}$. If $T$ has a left adjoint $S$ defined by a natural transformation $\kappa$, we will say that $(S, \kappa)$ is a functorial description of $\mathfrak{B}$. Notice that $S$ may just as well be considered as a functor of $\mathscr{C}$ into itself, that the notation $T$ for the natural injection of $\mathscr{C}^{\prime}$ in $\mathscr{C}$ may be dropped completely, and that $\kappa$ is then a natural transformation from $1_{\mathscr{C}}$ to $S$. This we will henceforward assume whenever we speak of a functorial description $(S, \kappa)$ of an injective structure $\mathfrak{B}$ of $\mathscr{C}$. It is then clear what we mean by a regular description of a regular injective structure $\mathfrak{B}$ of $\mathscr{C}$, and it follows immediately from Proposition 4 that any two regular descriptions of $\mathfrak{B}$ are equivalent.

Proposition 5. If the injective structures $\mathfrak{B}(\mathfrak{S}, \mathfrak{Q})$ and $\mathfrak{B}^{\prime}\left(\mathfrak{S}^{\prime}, \mathfrak{Q}^{\prime}\right)$ of $\mathscr{C}$ are described by $(S, \kappa)$ and $\left(S^{\prime}, \kappa^{\prime}\right)$, respectively, and if for each object $A$ of $\mathscr{C}$, there exists $\mu_{A}: S(A) \rightarrow S^{\prime}(A)$ such that $\kappa_{A}^{\prime}=\mu_{A} \kappa_{A}$, then $\mathfrak{B}$ is finer than $\mathfrak{V}^{\prime}$.

Proof. Let $f: A \rightarrow B$ be in $\mathfrak{S}$ and let $\phi: A \rightarrow Q^{\prime}$, where $Q^{\prime} \in \mathbb{Q}^{\prime}$. Then there exists $g: B \rightarrow S(A)$ such that $\kappa_{A}=g f$, and there exists $\psi: S^{\prime}(A) \rightarrow Q^{\prime}$ such that $\phi=\psi \kappa_{A}^{\prime}$, so that $\phi=\psi \mu_{A} \kappa_{A}=\left(\psi \mu_{A} g\right) f$. Therefore $f \in \Psi\left(\mathfrak{Q}^{\prime}\right)=\mathfrak{S}^{\prime}$.

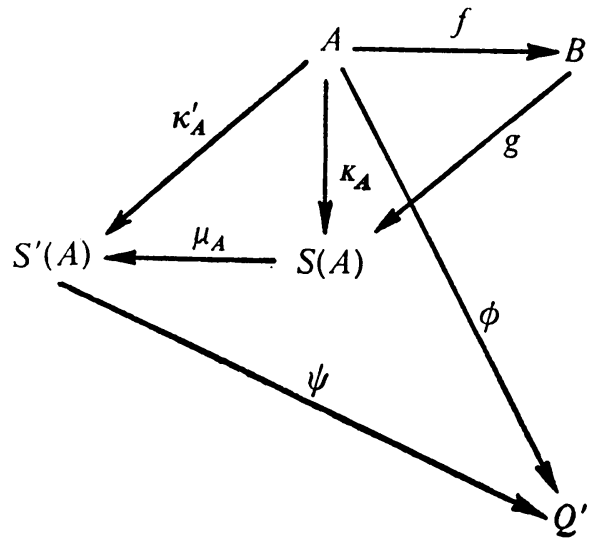


Now let $T: \mathscr{C}^{\prime} \rightarrow \mathscr{C}$ be any functor and assume that $T$ has a left adjoint system $(S, \kappa)$. Then by Proposition 2 of $\S 1,\left\{T\left(A^{\prime}\right)\right\}_{A^{\prime} \in \overline{\mathscr{C}}}$ is a basis of an injective structure $\mathfrak{B}$ of $\mathscr{C}$. We now generalize this situation. Let $\mathfrak{B}^{\prime}\left(\mathfrak{S}^{\prime}, \mathfrak{Q}^{\prime}\right)$ be an injective structure of $\mathscr{C}^{\prime}$. As above, $\mathfrak{Q}^{\prime}$ determines a complete subcategory $\mathscr{C}^{\prime \prime}$ of $\mathscr{C}^{\prime}$, and if $T^{\prime}$ denotes the natural injection of $\mathscr{C}^{\prime \prime}$ into $\mathscr{C}^{\prime}, T^{\prime}$ has a left adjoint system so that $T T^{\prime}$ has a left adjoint system which determines an injective structure $\mathfrak{B}(\mathfrak{S}, \mathfrak{Q})$ of $\mathscr{C}, T\left(\mathfrak{Q}^{\prime}\right)$ being a basis of injectives of $\mathfrak{B}$. We will say that $\mathfrak{B}$ is induced by $\mathfrak{B}^{\prime}$ through $T$. We notice that $\mathfrak{S}$ contains any $f: A \rightarrow B$ for which there exists $g: S(A) \rightarrow S(B)$ in $\mathcal{S}^{\prime}$ such that the diagram

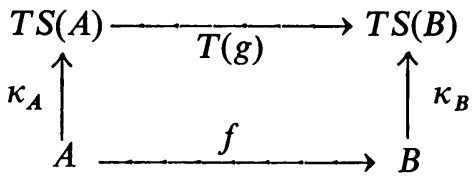

is commutative. If $\phi: A \rightarrow T\left(Q^{\prime}\right), Q^{\prime} \in Q^{\prime}$, there exists $\psi: S(A) \rightarrow Q^{\prime}$ such that $\phi=T(\psi) \kappa_{A}$, and then there exists $\chi: S(B) \rightarrow Q^{\prime}$ such that $\psi=\chi g$ so that

$$
\phi=T(\chi g) \kappa_{A}=T(\chi)\left(T(g) \kappa_{A}\right)=\left(T(\chi) \kappa_{B}\right) f .
$$

If $\kappa$ is a natural transformation defining a left adjoint functor $S$ of $T$, then what we have just seen is that $\mathfrak{S} \supseteq S^{-1}\left(\mathcal{S}^{\prime}\right)$. If, furthermore, $S$ is a regular left adjoint of $T$, then we have that $\mathfrak{S}=S^{-1}\left(\mathfrak{S}^{\prime}\right)$. If $f: A \rightarrow B$ is in $\mathfrak{S}$, and if $\phi: S(A) \rightarrow Q^{\prime} \in \mathfrak{Q}^{\prime}$, then there exists $\psi: B \rightarrow T\left(Q^{\prime}\right)$ such that $T(\phi) \kappa_{A}=\psi f$ and then there exists $\chi: S(B) \rightarrow Q^{\prime}$ such that $\psi=T(\chi) \kappa_{B}$ so that

$$
\begin{aligned}
T(\phi) \kappa_{A}= & T(\chi) \kappa_{B} f=T(\chi) T S(f) \kappa_{A}=T(\chi S(f)) \kappa_{A} . \\
T S(A) \longrightarrow T S(B) & T \kappa_{B} \\
\kappa_{A} \uparrow & f
\end{aligned}
$$

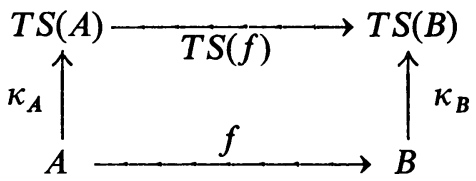

Therefore $\phi=\chi S(f)$.

It is then obvious that if $S$ has the property that a morphism $f$ in $\mathscr{C}$ is a monomorphism if and only if $S(f)$ is a monomorphism and if $\mathfrak{B}^{\prime}$ is the exact injective structure of $\mathscr{C}^{\prime}$, i.e., if $\mathfrak{S}^{\prime}$ consists of all the monomorphisms of $\mathscr{C}^{\prime}$, then $\mathfrak{B}$ is the exact structure of $\mathscr{C}$. (Given a ring homomorphism $\psi: R \rightarrow R^{\prime}$, if one applies this last statement to the functors $T=\operatorname{Hom}_{R}\left(R^{\prime}, \mathscr{C}_{R}\right)$ and $S$, the natural injection of $\mathscr{C}_{R}$, in $\mathscr{C}_{R}$, one obtains a well-known procf of the existence of enough injectives in a category of modules (see $[4$, p. 31]).)

We now consider an example. A ring homomorphism $\psi: R \rightarrow R^{\prime}$ will always apply unity element onto unity element. Given such a ring homomorphism, any module in $\mathscr{C}_{R}$. may be turned into a right $R$-module in a natural way and then any $R^{\prime}$-homomorphism $f^{\prime}: A^{\prime} \rightarrow B^{\prime}$ may be considered as an $R$-homomorphism 
of the corresponding right $R$-modules. This defines a functor $T$ from $\mathscr{C}_{R^{\prime}}$ to $\mathscr{C}_{R}$. If $A$ is any module in $\mathscr{C}_{R}$, then $A \otimes_{R} R^{\prime}$ may be turned into a right $R^{\prime}$-module $S(A)$ by defining $(a \otimes x) y=a \otimes(x y)$ for each $a \in A$ and each $x, y \in R^{\prime}$. Then, if $\kappa_{A}$ is that map from $A$ into $S(A)$ which maps each $a \in A$ onto $a \otimes 1,(S, \kappa)$ is a regular left adjoint system of $T$, as is well known. If $\mathfrak{B}^{\prime}\left(\mathbb{S}^{\prime}, \mathfrak{Q}^{\prime}\right)$ is an injective structure of $\mathscr{C}_{R^{\prime}}$, then, as we have seen above, $\mathfrak{B}^{\prime}$ induces an injective structure $\mathfrak{B}(\mathcal{S}, \mathfrak{Q})$ of $\mathscr{C}_{\mathbb{R}}$ through $T$, where $\subseteq$ is the class of all $R$-homomorphisms $f: A \rightarrow B$ in $\mathscr{C}_{R}$ such that that $S(f): S(A) \rightarrow S(B)$ is in $\mathcal{S}^{\prime}$ and where $\mathfrak{B}$ has a basis of injectives consisting of all $R^{\prime}$-modules in $\mathfrak{Q}^{\prime}$ considered as $R$-modules. We notice that if $N$ is an ideal of $R$ and if $\psi$ is the natural homomorphism of $R$ onto $R / R N$, then for each module $A$ in $\mathscr{C}_{R}, \kappa_{A}$ may be identified with the natural homomorphism of $A$ onto $A / A N$.

We notice that there are three possible dualizations of the preceding theory, obtained by replacing $\mathscr{C}$ or $\mathscr{C}^{\prime}$ or both by their duals.

\section{Radicals.}

Definition 1. A preradical $\mathfrak{r}$ of $\mathscr{C}_{R}$ is a function which assigns to each module $A$ of $\mathscr{C}_{\mathrm{R}}$ a submodule $\mathfrak{r}(A)$ of $A$ which is such that if $f: A \rightarrow B$, then $f$ maps $\mathfrak{r}(A)$ into $\mathfrak{r}(B)$.

A preradical of $\mathscr{C}_{R}$ may thus be considered as a functor from $\mathscr{C}_{R}$ to $\mathscr{C}_{R}$ or, more precisely, as a subfunctor of the identity functor of $\mathscr{C}_{R}$.

One may define a partial order relation on the class of all preredicals of $\mathscr{C}_{R}$ in the obvious way: if $\mathfrak{r}_{1}$ and $\mathfrak{r}_{2}$ are prereradicals of $\mathscr{C}_{R}, \mathfrak{r}_{1} \subseteq \mathfrak{r}_{2}$ if for any module $A$ of $\mathscr{C}_{R}, \mathfrak{r}_{1}(A) \subseteq \mathfrak{r}_{2}(A)$. Then the function $\mathfrak{r}_{0}$, which assigns to each module $A$ of $\mathscr{C}_{R}$ its 0-submodule, is the smallest preradical of $\mathscr{C}_{R}$, while the function $\mathfrak{r}_{I}$, which assigns to each module $A$ of $\mathscr{C}_{R} A$ itself, is the largest preradical of $\mathscr{C}_{R}$. Also, if $\left\{\mathfrak{r}_{i}\right\}_{i \in I}$ is any family of preradicals of $\mathscr{C}_{R}$, the functions which assign to each module $A$ of $\mathscr{C}_{R} \bigcap_{i \in I} \mathfrak{r}_{i}(A)$ and $\sum_{i \in I} \mathfrak{r}_{i}(A)$, respectively, are the greatest lower bound or intersection and the least upper bound or sum of the given preradicals, respectively. We will denote them by $\bigcap_{i \in I} \mathfrak{r}_{i}$ and $\sum_{i \in I} \mathfrak{r}_{i}$.

Given two preradicals $\mathfrak{r}_{1}$ and $\mathfrak{r}_{2}$ of $\mathscr{C}_{R}$, one can define their product $\mathfrak{r}_{2} \mathfrak{r}_{1}$ and their quotient $r_{1}: r_{2}$ to be the functions which, respectively, assign to each module $A$ of $\mathscr{C}_{R}$ the submodules $\mathfrak{r}_{2}\left(\mathfrak{r}_{1}(A)\right)$ and $\phi^{-1}\left(\mathfrak{r}_{2}\left(A / \mathfrak{r}_{1}(A)\right)\right)$ where $\phi$ is the natural homomorphism $A \rightarrow A / \mathfrak{r}_{1}(A)$. That these are preradicals of $\mathscr{C}_{R}$ is easy to verify.

Now if $\mathfrak{r}$ is a preradical of $\mathscr{C}_{R}$, we define the following sequence of preradicals of $\mathscr{C}_{R}$. We first define $\mathfrak{r}^{1}=\mathfrak{r}$, and then assuming that $\mathfrak{r}$ has been defined for all $\alpha<\beta$, we set $\mathfrak{x}^{\beta}=\mathfrak{r r}^{\beta-1}$ if $\beta$ is not a limit ordinal and $\mathfrak{r}^{\beta}=\bigcap_{\alpha<\beta} \mathfrak{r}$ if $\beta$ is a limit ordinal. Then the intersection $\hat{\mathfrak{r}}$ of all the $\mathfrak{r}^{\alpha}, \alpha$ running through the class of all ordinals, is a preradical of $\mathscr{C}_{R}$ which assigns to each module $A$ of $\mathscr{C}_{R}$ the largest submodule $B$ of $A$ for which $\mathfrak{r}(B)=B$. It is obvious that $\hat{\mathfrak{r}}$ is the largest preradical smaller than $\mathfrak{r}$ for which $\mathfrak{r} \hat{\mathfrak{r}}=\hat{\mathfrak{r}}$ or for which $\hat{\mathfrak{r}}^{2}=\hat{\mathfrak{x}}$.

Similarly, or really by duality, one may construct a smallest preradical $\overline{\mathfrak{r}}$ larger 
than $\mathfrak{r}$ for which $\overline{\mathfrak{r}}: \mathfrak{r}=\overline{\mathfrak{r}}$ or for which $\overline{\mathfrak{r}}: \overline{\mathfrak{r}}=\overline{\mathfrak{r}}$. This preradical $\overline{\mathfrak{r}}$ assigns to each module $A$ of $\mathscr{C}_{R}$ the smallest submodule $B$ of $A$ for which $\mathfrak{r}(A / B)=0$.

Definition 2. A preradical $\mathfrak{r}$ of $\mathscr{C}_{R}$ will be called a radical if $\overline{\mathfrak{r}}=\mathfrak{r}$, i.e., if for every module $A$ of $\mathscr{C}_{R}, \mathfrak{r}(A / \mathfrak{r}(A))=0$.

We notice that $\mathfrak{r}_{0}$ and $\mathfrak{r}_{I}$ are both radicals of $\mathscr{C}_{R}$ and that $\hat{\mathfrak{r}}_{0}=\mathfrak{r}_{0}$ and $\hat{\mathfrak{r}}_{I}=\mathfrak{r}_{I}$.

LEMMA 1. If $\mathfrak{r}$ is a radical of $\mathscr{C}_{R}$ and if $A \subseteq \mathfrak{r}(B)$, then $\mathfrak{r}(B / A)=\mathfrak{r}(B) / A$.

Proof. The natural homomorphism of $B$ onto $B / A$ maps $\mathfrak{r}(B)$ into $\mathfrak{r}(B / A)$ so that $\mathfrak{r}(B) / A \subseteq \mathfrak{r}(B / A)$. The natural homomorphism of $B / A$ onto $B / \mathfrak{r}(B)$ maps $\mathfrak{r}(B / A)$ into $\mathfrak{r}(B / \mathfrak{r}(B))=0$ so that $\mathfrak{r}(B / A) \subseteq \mathfrak{r}(B) / A$.

PRoposition 1. (a) If $\mathfrak{r}_{1}$ and $\mathfrak{r}_{2}$ are radicals of $\mathscr{C}_{R}$, then so is $\mathfrak{r}_{1} \mathfrak{r}_{2}$.

(b) If $\left\{\mathfrak{r}_{i}\right\}_{i \in I}$ is a family of radicals of $\mathscr{C}_{R}$, then so is $\bigcap_{i \in I} \mathfrak{r}_{i}$.

(c) If $\mathfrak{r}$ is a radical of $\mathscr{C}_{R}$, then so is $\hat{\mathfrak{r}}$.

Proof. Let $A$ be any module in $\mathscr{C}_{R}$.

(a) By Lemma $1, \mathfrak{r}_{2}\left(A / \mathfrak{r}_{1} \mathfrak{r}_{2}(A)\right)=\mathfrak{r}_{2}(A) / \mathfrak{r}_{1} \mathfrak{r}_{2}(A)$ so that

$$
\mathfrak{r}_{1} \mathfrak{r}_{2}\left(A / \mathfrak{r}_{1} \mathfrak{r}_{2}(A)\right)=\mathfrak{r}_{1}\left(\mathfrak{r}_{2}\left(A / \mathfrak{r}_{1} \mathfrak{r}_{2}(A)\right)\right)=\mathfrak{r}_{1}\left(\mathfrak{r}_{2}(A) / \mathfrak{r}_{1}\left(\mathfrak{r}_{2}(A)\right)\right)=0 .
$$

(b) Let $\mathfrak{r}=\bigcap_{i \in I} \mathfrak{r}_{i}$. By Lemma 1 , for each $i \in I, \mathfrak{r}_{i}(A / \mathfrak{r}(A))=\mathfrak{r}_{i}(A) / \mathfrak{r}(A)$ so that

$$
\begin{aligned}
\mathfrak{r}(A / \mathfrak{r}(A)) & =\bigcap_{i \in I} \mathfrak{r}_{i}(A / \mathfrak{r}(A))=\bigcap_{\in \in I} \mathfrak{r}_{i}(A) / \mathfrak{r}(A) \\
& =\left(\bigcap_{\in} \mathfrak{r}_{I}(A)\right) / \mathfrak{r}(A)=0 .
\end{aligned}
$$

(c) follows from (a), (b) and the definition of $\hat{\mathfrak{r}}$.

Definition 3. If $\mathfrak{r}$ is a preradical of $\mathscr{C}_{R}$ and if $A \subseteq B$, then we will say that $A$ is $\mathfrak{r}$-dense in $B$ and write $A \leqq_{\mathfrak{r}} B$ if $\mathfrak{r}(B / A)=B / A$. If $\mathfrak{r}(A)=0$, then we will say that $A$ is $\mathfrak{r}$-separated.

Proposition 2. If $\mathfrak{r}$ is a preradical of $\mathscr{C}_{R}$, then

$$
A \subseteq B \subseteq C \& A \leqq{ }_{\mathfrak{r}} C \Rightarrow B \leqq{ }_{\mathfrak{r}} C,
$$

and if $\mathfrak{r}$ is a radical,

$$
A \leqq_{\mathfrak{r}} B \& B \varliminf_{\mathfrak{r}} C \Rightarrow A \leqq{ }_{\mathfrak{r}} C .
$$

Proof. To prove the first part, we need only remark that the natural homomorphism of $C / A$ onto $C / B$ maps $C / A=\mathfrak{r}(C / A)$ into $\mathfrak{r}(C / B)$ so that $\mathfrak{r}(C / B)=C / B$.

To prove the second part, we notice that $B / A=\mathfrak{r}(B / A) \subseteq \mathfrak{r}(C / A)$ so that by Lemma 1, $\mathfrak{r}(C / A) /(B / A)=\mathfrak{r}((C / A) /(B / A))$. However, $(C / A) /(B / A) \cong C / B=\mathfrak{r}(C / B)$, so that $\mathfrak{r}((C / A) /(B / A))=(C / A) /(B / A)$ and, therefore, $\mathfrak{r}(C / A)=C / A$, i.e., $A \leqq_{\mathfrak{r}} C$.

Let $\mathfrak{B}(\mathfrak{S}, \mathfrak{Q})$ be an injective structure of $\mathscr{C}_{R}$. If $A$ is any module in $\mathscr{C}_{R}$, there exists $\phi: A \rightarrow Q$ in $\mathfrak{S}$, where $Q \in \mathfrak{Q}$. If $\psi: A \rightarrow B$ is in $\mathfrak{S}$, there exists $\chi: B \rightarrow Q$ such that $\phi=\chi \psi$ so that $\operatorname{ker} \psi \subseteq \operatorname{ker} \phi$. 


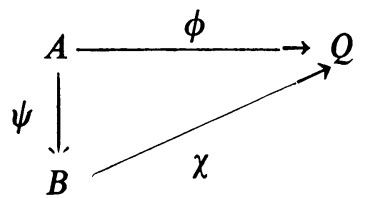

Thus $\operatorname{ker} \phi$ is the largest kernel of a homomorphism in $\mathfrak{S}$ defined on $A$. We denote it by $\mathfrak{r}_{\mathfrak{B}}(A)$ and show that the function $\mathfrak{r}_{\mathfrak{B}}$ is a radical of $\mathscr{C}_{\mathbb{R}}$. If $f: A \rightarrow B$, let $\phi: A \rightarrow Q$ and $\psi: B \rightarrow Q^{\prime}$ be homomorphisms in $\subseteq$, where $Q$ and $Q^{\prime}$ are in $Q$. Then there exists $g: Q \rightarrow Q^{\prime}$ such that $\psi f=g \phi$ so that $f$ maps $\operatorname{ker} \phi=\mathfrak{r}_{\mathfrak{B}}(A)$ into $\operatorname{ker} \psi=\mathfrak{r}_{\mathfrak{B}}(B)$ and therefore $\mathfrak{r}_{\mathfrak{B}}$ is a preradical. Furthermore, $\phi=k h$, where $h$ is the natural homomorphism of $A$ onto $A / \mathfrak{r}_{\mathfrak{g}}(A)$ and $k$ is a monomorphism. By B3, $k \in \mathfrak{S}$ so that $\mathfrak{r}_{\mathfrak{2}}\left(A / \mathfrak{r}_{\mathfrak{2}}(A)\right)=0$ and therefore $\mathfrak{r}_{\mathfrak{B}}$ is a radical. It is obvious that every module in $\mathfrak{Q}$ is $\mathfrak{r}_{\mathfrak{B}}$-separated. We notice the following example: by Proposition 3 of $\S 1$, the set of all inequivalent simple modules in $\mathscr{C}_{R}$ is a subbasis of injectives of an injective structure $\mathfrak{B}$ of $\mathscr{C}_{R}$, and for any module $A$ in $\mathscr{C}_{R}$ it is obvious that $\mathfrak{r}_{\mathfrak{B}}(A)$ is the radical of $A$ in the sense of Bourbaki [2].

The following three statements are easy to verify.

(1) Given two injective structures $\mathfrak{V}_{1}$ and $\mathfrak{V}_{2}$ of $\mathscr{C}_{R}$, if $\mathfrak{V}_{1}$ is finer than $\mathfrak{V}_{2}$, then $\mathfrak{r}_{\mathfrak{B}_{1}} \subseteq \mathfrak{r}_{\mathfrak{B}_{2}}$.

(2) The coarsest and finest injective structures of $\mathscr{C}_{R}$ respectively define the largest and smallest radicals of $\mathscr{C}_{R}$.

(3) From the proof of Proposition 4 of $\S 1$ it follows that if $\left\{\mathfrak{B}_{i}\right\}_{i \in I}$ is a family of injective structures of $\mathscr{C}_{R}$ and if $\mathfrak{B}=\sup _{i \in I} \mathfrak{B}_{i}$, then $\mathfrak{r}_{\mathfrak{B}}=\bigcap_{i \in I} \mathfrak{r}_{\mathfrak{B}_{i}}$.

Proposition 3. If $\mathfrak{r}$ is a radical of $\mathscr{C}_{R}$, then the class of all $\mathfrak{r}$-separated modules is the class of injectives of a regular injective structure $\mathfrak{B}$ of $\mathscr{C}_{R}$. It is the finest injective structure of $\mathscr{C}_{R}$ defining the radical $\mathfrak{r}$.

Proof. Let $A$ be any module in $\mathscr{C}_{R}$ and let $\phi$ be the natural homomorphism of $A$ onto $A / \mathfrak{r}(A)$. If $f: A \rightarrow B$, where $B$ is $\mathfrak{r}$-separated, then $\mathfrak{r}(A) \subseteq \operatorname{ker} f$ so that there exists one and only one $g: A / \mathfrak{r}(A) \rightarrow B$ such that $f=g \phi$. From Proposition 4 of $\S 1$, we conclude immediately that the $\mathfrak{r}$-separated modules are the injectives of a regular injective structure $\mathfrak{B}$ of $\mathscr{C}_{R}$ obviously defining the radical $\mathfrak{r}$. If $\mathfrak{B}^{\prime}\left(\mathfrak{S}^{\prime}, \mathfrak{Q}^{\prime}\right)$ is any injective structure of $\mathscr{C}_{R}$ defining the radical $\mathfrak{r}$, then, as was stated above, every module in $Q^{\prime}$ is $\mathfrak{r}$-separated so that $\mathfrak{B}^{\prime}$ is coarser than $\mathfrak{B}$.

Proposition 4. If $\mathfrak{B}(\mathfrak{S}, \mathfrak{Q})$ is an injective structure of $\mathscr{C}_{R}$ defining the radical $\mathfrak{r}$ and if $\phi: A \rightarrow B$, then $\operatorname{im} \phi \varliminf_{\mathfrak{r}} B$ if and only if, for any two homomorphisms $g$ and $g^{\prime}$ from $B$ to some $Q \in \mathbb{Q}, g \phi=g^{\prime} \phi$ implies that $g=g^{\prime}$.

Proof. Set $D=B / \operatorname{im} \phi$ and let $\psi$ denote the natural homomorphism of $B$ onto $D$. Assume first of all that im $\phi \leqq_{\mathfrak{r}} B$. If $g, g^{\prime}: B \rightarrow Q$, where $Q \in \mathbb{Q}$, and if $g \phi=g^{\prime} \phi$, i.e., $\left(g-g^{\prime}\right) \phi=0$, then there exists $h: D \rightarrow Q$ such that $g-g^{\prime}=h \psi$. But since $Q$ is 
$\mathfrak{r}$-separated, $h$ maps $D=\mathfrak{r}(D)$ into $\mathfrak{r}(Q)=0$ so that $h=0$ and therefore $g=g^{\prime}$. Conversely, assume that $g \phi=g^{\prime} \phi$, where $g, g^{\prime}: B \rightarrow Q, Q \in \mathbb{Q}$, implies that $g=g^{\prime}$. Then there exists $\chi: D \rightarrow Q$ in $\mathfrak{S}$, where $Q \in \mathfrak{Q}$, and as we know, $\operatorname{ker} \chi=\mathfrak{r}(D)$. Obviously, $\chi \psi \phi=0$ so that by our hypothesis $\chi \psi=0$ and since $\psi$ is an epimorphism, $\chi=0$ and therefore $D=\mathfrak{r}(D)$, i.e., $\operatorname{im} \phi \varliminf_{\mathfrak{r}} B$.

Notice that by Proposition $3, \mathfrak{Q}$ may be taken to be the class of all $\mathfrak{r}$-separate modules in Proposition 4.

THEOREM 1. If $\mathfrak{B}(\mathfrak{S}, \mathfrak{Q})$ is an injective structure of $\mathscr{C}_{R}$, then the class $\mathfrak{Q}^{*}$ of all modules $A$ in $\mathscr{C}_{R}$ for which there exists a monomorphism $\phi: A \rightarrow Q$ in $\mathfrak{S}$, where $Q \in \mathbb{Q}$ and where $\hat{\mathfrak{r}}_{\mathfrak{B}}(Q / \mathrm{im} \phi)=0$, is the class of injectives of the coarsest regular injective structure finer than $\mathfrak{B}$. Furthermore $\mathfrak{r}_{\mathfrak{B}^{*}}=\mathfrak{r}_{\mathfrak{B}}$.

Proof. Let $\mathfrak{S}_{1}$ be the class of all homomorphisms $\psi: C \rightarrow D$ in $\mathfrak{S}$ such that $\operatorname{im} \psi \leqq_{\mathfrak{r}_{\mathfrak{B}}} D$. Given such a homomorphism $\psi$ and given $f: C \rightarrow A$, where $A \in \mathbb{Q}^{*}$, we show that there exists a unique $g: D \rightarrow A$ such that $f=g \psi$. The uniqueness of $g$ follows immediately from Proposition 4. There exists $\phi: A \rightarrow Q$ in $\mathfrak{S}$, where $Q \in \mathfrak{Q}$ and $\hat{\mathfrak{r}}_{\mathfrak{B}}(Q / \operatorname{im} \phi)=0$. Since $Q \in \mathfrak{Q}$ and $\psi \in \mathfrak{S}$, there exists $k: D \rightarrow Q$ such that $\phi f=k \psi$ and $k$ induces $h: D / \operatorname{im} \psi \rightarrow Q / \operatorname{im} \phi$.

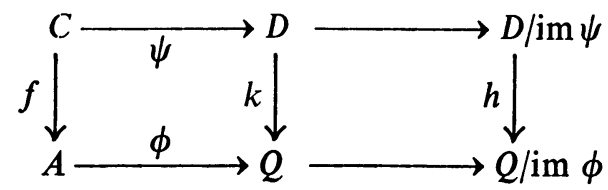

Then $h$ must map $D / \operatorname{im} \psi=\mathfrak{r}_{\mathfrak{B}}(D / \operatorname{im} \psi)=\hat{\mathfrak{r}}_{\mathfrak{B}}(D / \operatorname{im} \psi)$ into $\hat{\mathfrak{r}}_{\mathfrak{B}}(Q / \operatorname{im} \phi)=0$ so that $h=0$, and therefore there exists $g: D \rightarrow A$ such that $k=\phi g$. Then $\phi f=k \psi=\phi g \psi$, and since $\phi$ is a monomorphism, $f=g \psi$.

Now, if $A$ is any module in $\mathscr{C}_{R}$, we show that there exists $\phi^{*}: A \rightarrow A^{*}$ in $\mathfrak{S}_{1}$, where $A^{*} \in Q^{*}$ and where $\operatorname{ker} \phi^{*}=\mathfrak{r}_{\mathfrak{B}}(A)$ so that, by Proposition 4 of $\S 1, \mathfrak{Q}^{*}$ is the class of injectives of a regular injective structure $\mathfrak{B}^{*}$ of $\mathscr{C}_{R}$ defining the radical $\mathfrak{r}_{\mathfrak{V}}$. We know that there exists $\phi: A \rightarrow Q$ in $\mathfrak{S}$, where $Q \in \mathbb{Q}$. Let $\chi$ denote the natural homomorphism of $Q$ onto $Q / \operatorname{im} \phi$ and let $A^{*}=\chi^{-1}\left(\hat{r_{3}}(Q / \operatorname{im} \phi)\right)$. Then, $\phi=\psi \phi^{*}$, where $\psi$ is the natural injection of $A^{*}$ into $Q$, and obviously, $\operatorname{ker} \phi^{*}=\mathfrak{r}_{\mathfrak{B}}(A)$. By B3, $\phi^{*} \in \mathfrak{S}$. Furthermore,

$$
\hat{\mathfrak{r}}_{\mathfrak{B}}\left(A^{*} / \operatorname{im} \phi^{*}\right)=\hat{\mathfrak{r}}_{\mathfrak{B}}\left(\hat{\mathfrak{r}}_{\mathfrak{B}}(Q / \operatorname{im} \phi)\right)=\hat{\mathfrak{r}}_{\mathfrak{B}}(Q / \operatorname{imp} \phi)=A^{*} / \operatorname{im} \phi^{*}
$$

so that $\phi^{*} \in \mathfrak{S}_{1}$. Now we need to show that $\psi \in S$. Let $f: A^{*} \rightarrow Q^{\prime} \in \mathbb{Q}$.

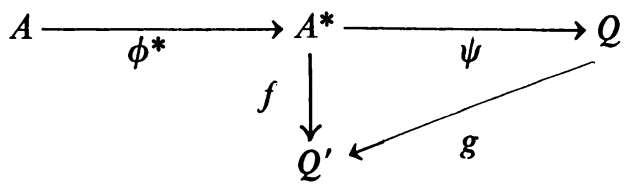


There exists $g: Q \rightarrow Q^{\prime}$ such that $f \phi^{*}=g \phi=g \psi \phi^{*}$. Since $\mathfrak{r}_{\mathfrak{w}}\left(A / \mathrm{im} \phi^{*}\right)=A / \operatorname{im} \phi^{*}$ and since $Q^{\prime} \in \mathfrak{Q}$, by Proposition 4, $f=g \psi$. But also, since

$$
\hat{\mathfrak{r}}_{\mathfrak{B}}\left(Q / A^{*}\right) \cong \hat{\mathfrak{r}}_{\mathfrak{B}}\left((Q / \operatorname{im} \phi) / \hat{\mathfrak{r}}_{\mathfrak{B}}(Q / \operatorname{im} \phi)\right)=0,
$$

we have that $A^{*} \in \mathfrak{Q}^{*}$. It is obvious that $\mathfrak{B}^{*}$ is finer than $\mathfrak{B}$.

Now, if $\mathfrak{B}^{\prime}\left(\mathfrak{S}^{\prime}, \mathfrak{Q}^{\prime}\right)$ is a regular iniective structure of $\mathscr{C}_{R}$ finer than $\mathfrak{B}$. and if $\widetilde{S}_{1}^{\prime}$ is the class of all morphisms $\psi^{\prime}: C^{\prime} \rightarrow D^{\prime}$ in $\widetilde{S}^{\prime}$ for which $\mathfrak{r}_{\mathfrak{B}^{\prime}}\left(D^{\prime} / \operatorname{im} \psi^{\prime}\right)=D^{\prime} / \operatorname{im} \psi^{\prime}$, since $\mathfrak{r}_{\mathfrak{B}^{\prime}} \subseteq \mathfrak{r}_{\mathfrak{B}}$, by Proposition $4, \mathfrak{S}_{1}^{\prime} \subseteq \mathfrak{S}_{1}$ so that obviously $\mathfrak{Q}^{\prime}=\Phi\left(\mathfrak{S}_{1}^{\prime}\right) \supseteq \Phi\left(\mathfrak{S}_{1}\right)=\mathfrak{Q}^{*}$, i.e., $\mathfrak{V}^{\prime}$ is finer than $\mathfrak{B}^{*}$.

5. The ring homomorphism defined by a regular injective structure of $\mathscr{C}_{R}$. Let $\mathfrak{B}(S, \mathfrak{Q})$ be a regular injective structure of $\mathscr{C}_{R}$ and let $(S, \kappa)$ be a regular functorial description of $\mathfrak{B}$. If $A$ is any module in $\mathscr{C}_{R}$ and if $a \in A$, then we will denote by $\phi_{a}$ the only $R$-homomorphism from $R$ to $A$ that maps 1 onto $a$. This is a notation that we will adopt throughout this paper. If $Q \in \mathbb{Q}$, then for each $x \in Q$ there exists a unique homomorphism $\bar{\phi}_{x}: S(R) \rightarrow Q$ such that $\phi_{x}=\bar{\phi}_{x} \kappa_{R}$.

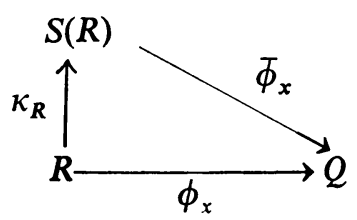

We define a product of the type $Q \times S(R) \rightarrow Q$ as follows: $x y=\Phi_{x}(y)$ for each $x \in Q$ and each $y \in S(R)$. Then, we have the following:

(1) If $x \in Q$ and $y, y^{\prime} \in S(R)$,

$$
x\left(y+y^{\prime}\right)=\Phi_{x}\left(y+y^{\prime}\right)=\Phi_{x}(y)+\Phi_{x}\left(y^{\prime}\right)=x y+x y^{\prime} .
$$

(2) If $x, x^{\prime} \in Q$ and $r \in R$,

$$
\left(\phi_{x}+\phi_{x^{\prime}}\right)(r)=\phi_{x}(r)+\phi_{x^{\prime}}(r)=x r+x^{\prime} r=\left(x+x^{\prime}\right) r=\phi_{x+x^{\prime}}(r)
$$

so that $\phi_{x}+\phi_{x^{\prime}}=\phi_{x+x^{\prime}}$. Therefore

$$
\left(\Phi_{x}+\bar{\phi}_{x^{\prime}}\right) \kappa_{R}=\Phi_{x} \kappa_{R}+\bar{\phi}_{x^{\prime}} \kappa_{R}=\phi_{x}+\phi_{x^{\prime}}=\phi_{x+x^{\prime}}=\Phi_{x+x^{\prime}} \kappa_{R}
$$

so that $\phi_{x}+\phi_{x^{\prime}}=\bar{\phi}_{x+x^{\prime}}$. Then, for any $y \in S(R)$,

$$
\left(x+x^{\prime}\right) y=\bar{\phi}_{x+x^{\prime}}(y)=\left(\Phi_{x}+\bar{\phi}_{x^{\prime}}\right)(y)=\Phi_{x}(y)+\bar{\phi}_{x}(y)=x y+x^{\prime} y .
$$

(3) If $x \in Q, y \in S(R)$ and $r \in R$,

$$
\begin{aligned}
\phi_{x} \Phi_{y} \kappa_{R}(r) & =\Phi_{x} \phi_{y}(r)=\phi_{x}(y r)=\left(\Phi_{x}(y)\right) r \\
& =(x y) r=\phi_{x y}(r)=\phi_{x y} \kappa_{R}(r)
\end{aligned}
$$

so that $\bar{\phi}_{x} \bar{\phi}_{y}=\bar{\phi}_{x y}$. Then, for any $y^{\prime} \in S(R)$, 


$$
(x y) y^{\prime}=\Phi_{x y}\left(y^{\prime}\right)=\Phi_{x} \Phi_{y}\left(y^{\prime}\right)=\Phi_{x}\left(y y^{\prime}\right)=x\left(y y^{\prime}\right) .
$$

This shows that $S(R)$ may be turned into a ring over which each module in $\mathfrak{Q}$ is a right module. Let us show that $\kappa_{R}$ is a ring homomorphism mapping unity element onto unity element and that each module in $\mathfrak{Q}$ is a unitary module over $S(R)$. If $r, r^{\prime} \in R$,

$$
\kappa_{R}(r) \kappa_{R}\left(r^{\prime}\right)=\bar{\phi}_{\kappa_{R}(r)} \kappa_{R}\left(r^{\prime}\right)=\phi_{\kappa_{R}(r)}\left(r^{\prime}\right)=\kappa_{R}(r) r^{\prime}=\kappa_{R}\left(r r^{\prime}\right) .
$$

If $Q \in \mathbb{Q}$ and $x \in Q$,

$$
x \kappa_{R}(1)=\bar{\phi}_{x} \kappa_{R}(1)=\phi_{x}(1)=x 1=x \text {. }
$$

If $r \in R$,

$$
\kappa_{R}(r)=\kappa_{R}(1 r)=\kappa_{R}(1) \kappa_{R}(r)=\bar{\phi}_{\kappa R(1)} \kappa_{R}(r)
$$

so that $1_{S(R)}=\Phi_{\kappa_{R}(1)}$ and then, for any $y \in S(R)$,

$$
\kappa_{R}(1) y=\bar{\phi}_{\kappa_{R}(1)}(y)=y \text {. }
$$

We notice that for any $Q \in \mathbb{Q}$, the $S(R)$-module structure we have just defined on $Q$ induces the original $R$-module structure on $Q$, for if $x \in Q$ and $r \in R$,

$$
x \kappa_{R}(r)=\bar{\phi}_{x} \kappa_{R}(r)=\phi_{x}(r)=x r .
$$

From the fact that $\kappa_{R}$ is a ring homomorphism, it follows, by Proposition 3 of $\S 3$, that if $\mathfrak{r}$ is a radical of $\mathscr{C}_{R}$, then $\mathfrak{r}(R)$ is a two-sided ideal of $R$.

LEMMA 1. If $f: Q \rightarrow Q^{\prime}$ is an $R$-homomorphism, where $Q, Q^{\prime} \in \mathbb{Q}$, and if one turns $Q$ and $Q^{\prime}$ into $S(R)$-modules as above, then $f$ is an $S(R)$-homomorphism.

Proof. If $x \in Q$ and $r \in R$,

$$
\phi_{f(x)}(r)=f(x) \cdot r=f(x r)=f \phi_{x}(r)
$$

so that $\phi_{f(x)}=f \phi_{x}$, i.e., $\Phi_{f(x)} \kappa_{R}=f \bar{\phi}_{x} \kappa_{R}$, and therefore $\bar{\phi}_{f(x)}=f \bar{\phi}_{x}$. Then if $y \in S(R)$,

$$
f(x y)=f \bar{\phi}_{x}(y)=\bar{\phi}_{f(x)}(y)=f(x) y .
$$

CoRollary. If $\mathscr{C}^{\prime}$ is the complete subcategory of $\mathscr{C}_{S(R)}$ whose objects are the modules in $\mathfrak{Q}$, turned into right $S(R)$-modules as above, then $S$ may be considered as a functor from $\mathscr{C}_{R}$ to $\mathscr{C}^{\prime}$ and, as such, it is a regular left adjoint of the natural injection of $\mathscr{C}^{\prime}$ into $\mathscr{C}_{R}$.

THEOREM 1. The class of all modules in $\mathfrak{Q}$ turned into right $S(R)$-modules as above is the class of injectives of a regular injective structure of $\mathscr{C}_{S(R)}$ whose class of proper maps is contained in the class of all $S(R)$-homomorphisms $f: A \rightarrow B$ that are in $\mathfrak{S}$ when considered as $R$-homomorphisms.

Proof. If $A$ is any $S(R)$-module and if $f: A \rightarrow Q$ is an $S(R)$-homomorphism, then there exists a unique $R$-homomorphism $g: S(A) \rightarrow Q$ such that $f=g \kappa_{A}$, and 
by Lemma $1, g$ is an $S(R)$-homomorphism. If we can show that $\kappa_{A}$ is an $S(R)$ homomorphism, the first part of this proposition will follow from Proposition 4 of $\S 1$. Let $a \in A$ and consider the two functions $\sigma$ and $\tau$ from $S(R)$ to $S(A)$ defined as follows:

$$
\sigma(y)=\kappa_{A}(a y), \quad \tau(y)=\kappa_{A}(a) \cdot y,
$$

for each $y \in S(R)$. It is obvious that $\sigma$ and $\tau$ are $R$-homomorphisms. Then, for each $r \in R$,

$$
\begin{aligned}
\sigma \kappa_{R}(r) & =\kappa_{A}\left(a \kappa_{R}(r)\right)=\kappa_{A}\left(a \kappa_{R}(1 \cdot r)\right)=\kappa_{A}\left(a \kappa_{R}(1) r\right) \\
& =\kappa_{A}(a r)=\kappa_{A}(a) r=\kappa_{A}(a) \kappa_{R}(r)=\tau \kappa_{R}(r)
\end{aligned}
$$

so that $\sigma=\tau$, and therefore $\kappa_{A}$ is an $S(R)$-homomorphism. Now let $f: A \rightarrow B$ be an $S(R)$-homomorphism which is proper with respect to this injective structure of $\mathscr{C}_{S(R)}$. Then there exists an $S(R)$-homomorphism $g: B \rightarrow S(A)$ such that $\kappa_{A}=g f$. Then, by B3, considered as an $R$-homomorphism, $f \in \mathfrak{S}$.

THEOREM 2. Under the conditions of Theorem 1, the following three statements are equivalent:

(1) The functors $S$ and $\mathscr{C}_{R} \otimes_{R} S(R)$ are equivalent.

(2) If $A$ is an $S(R)$-module, then $\mathfrak{r}(A)=0$.

(3) Every $S(R)$-module is an $R$-module in $\mathfrak{Q}$, i.e., the regular injective structure of $\mathscr{C}_{S(R)}$ whose existence is asserted in Theorem 1 is the finest injective structure of $\mathscr{C}_{S(R)}$.

Proof. That (1) implies (2) is obvious. Assume that (2) holds and let $A$ be a module in $\mathscr{C}_{S(R)}$. Then $\operatorname{ker} \kappa_{A}=\mathfrak{r}(A)=0$, and since $\kappa_{A}$ is an $S(R)$-homomorphism, $S(A) / \kappa_{A}(A)$ is an $S(R)$-module so that $S(A) / \kappa_{A}(A)=\mathfrak{r}\left(S(A) / \kappa_{A}(A)\right)=0$. Therefore $\kappa_{A}$ is an isomorphism and $A \in \mathbb{Q}$.

Finally, assume that (3) holds. Let $\mu$ denote the canonical $R$-homomorphism from $A$ to $A \otimes_{R} S(R)$. Then $A \otimes_{R} S(R)$ can be turned into an $S(R)$-module in a natural way and there exists a unique $S(R)$-homomorphism $\phi_{A}: A \otimes_{R} S(R) \rightarrow S(A)$

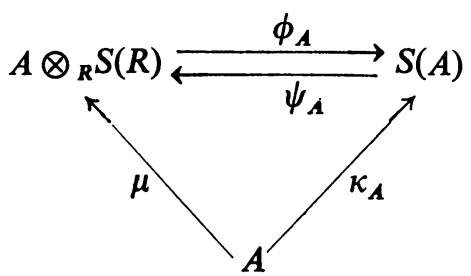

such that $\kappa_{A}=\phi_{A} \mu$. Then, since $A \otimes_{R} S(R)$, as an $R$-module, is in $\mathcal{Q}$ and $\kappa_{A} \in S$, there exists a unique $R$-homomorphism $\psi_{A}: S(A) \rightarrow A \otimes_{R} S(R)$ such that $\mu=\psi_{A} \kappa_{A}$ and by Lemma $1, \psi_{A}$ is an $S(R)$-homomorphism. We then have that 


$$
1_{(S A)} \kappa_{A}=\kappa_{A}=\psi_{A} \phi_{A} \kappa_{A}
$$

and therefore $1_{S(A)}=\phi_{A} \psi_{A}$. We also have that

$$
1_{A} \otimes_{R} S(R)=\mu=\psi_{A} \phi_{A} \mu
$$

and therefore $1_{A} \otimes_{R} S(R)=\psi_{A} \phi_{A}$. Thus $\mathscr{C}_{R} \otimes_{R} S(R)$ and $S$ are equivalent.

Now we show by an example that the three equivalent statements in Theorem 2 do not always hold. Let $R$ be the ring of ordinary integers and let $p$ be a prime of $R$. Then the set of all cyclic modules of orders $p^{n}, n=1,2,3, \cdots$, is a subbasis of injectives of a regular injective structure $\mathfrak{B}(\mathfrak{S}, \mathfrak{Q})$ of $\mathscr{C}_{R}(10)$, if $\mathfrak{r}$ denotes the radical of $\mathscr{C}_{R}$ defined by $\mathfrak{B}$, for any module $A, \mathfrak{r}(A)=\bigcap_{n=1} p^{n} A$, and if $(S, \kappa)$ is a functorial description of $\mathfrak{B}, S(R)$ is the ring of $p$-adic integers. Then, if $A$ is an $R$-module of type $p^{\infty}, \mathfrak{r}(A)=A \neq 0$ and $A$ is an $S(R)$-module.

THEOREM 3. If $\psi: R \rightarrow R^{\prime}$ is a ring homomorphism and if $R^{\prime}$, turned into a right $R$-module in the natural way, is $\mathfrak{r}_{\mathfrak{B}^{3}}$-separated and contains an $R$-submodule $C \in \mathbb{Q}$ containing $\psi(R)$, in particular, if $R^{\prime} \in \mathbb{Q}$, then there exists a unique ring homomorphism $\chi: S(R) \rightarrow R^{\prime}$ such that $\psi=\chi \kappa_{R}$.

Proof. There exists a right $R$-homomorphism $\chi: S(R) \rightarrow C$ such that $\psi=\chi \kappa_{R}$, and since $R^{\prime}$ is $\mathfrak{r}_{\mathfrak{B}}$-separated, $\chi$ is the only right $R$-homomorphism from $S(R)$ to $R^{\prime}$ with this property. Let $y$ be an arbitrary element of $S(R)$ and consider the two maps $\sigma$ and $\tau$ from $S(R)$ to $R^{\prime}$ defined as follows: $\sigma\left(y^{\prime}\right)=\chi(y) \chi\left(y^{\prime}\right)$, $\tau\left(y^{\prime}\right)=\chi\left(y y^{\prime}\right)$ for each $y^{\prime} \in S(R)$. It is obvious that $\sigma$ and $\tau$ are right $R$-homomorphisms. But for any $r \in R$,

$$
\sigma \kappa_{R}(r)=\chi(y) \chi \kappa_{R}(r)=\chi(y) \psi(r)=\chi(y) r=\chi(y r)=\chi\left(y \kappa_{R}(r)\right)=\tau \kappa_{R}(r)
$$

so that $\sigma=\tau$, and therefore $\chi$ is a ring homomorphism. If $\chi^{\prime}$ is any ring homomorphism from $S(R)$ to $R^{\prime}$ such that $f=\chi^{\prime} \kappa_{R}$, then for any $y \in S(R)$ and any $r \in R$,

$$
\chi^{\prime}(y r)=\chi^{\prime}\left(y \kappa_{R}(r)\right)=\chi^{\prime}(y) \chi^{\prime} \kappa_{R}(r)=\chi^{\prime}(y) \psi(r)=\chi^{\prime}(y) r
$$

so that $\chi^{\prime}$ is a right $R$-homomorphism and therefore $\chi^{\prime}=\chi$.

We now define, for any module $A$ in $\mathscr{C}_{R}$, a canonical correspondence, always denoted by $\rho$, between the $R$-submodules of $A$ and the $S(R)$-submodules of $S(A)$ that generalizes the well-known correspondence between the ideals of a commutative ring and the ideals of one of its rings of quotients and that also generalizes the correspondence between the ideals of, say, the ring of ordinary integers and the ideals of one of its $p$-adic completions.

If $B$ is an $R$-submodule of $A$ and if $\pi$ denotes the natural homomorphism of $A$ onto $A / B$, then we define $\rho(B)$ to be the kernel of $S(\pi): S(A) \rightarrow S(A / B)$. Then by Lemma $1, \rho(B)$ is an $S(R)$-submodule of $S(A)$ obviously containing $\kappa_{A}(B)$. Furthermore, $\rho$ has the following three properties: 
(1) $\rho(B)$ is the smallest $R$-submodule of $S(A)$ containing $\kappa_{A}(B)$ such that $S(R) / \rho(B)$ is $\mathfrak{r}_{\mathfrak{2}}$-separated.

Proof. That $S(A) / \rho(B)$ is $\mathfrak{r}_{\mathfrak{B}}$-separated is immediate since $S(\pi)$ is a homomorphism into the $\mathfrak{r}_{\mathfrak{B}}$-separated module $S(A / B)$. Let $C$ be an $R$-submodule of $S(A)$ containing $\kappa_{A}(B)$ and such that $S(A) / C$ is $\mathfrak{r}_{2}$-separated, and let $\mu$ be the natural homomorphism of $S(A)$ onto $S(A) / C$. Since $\kappa_{A}(B) \subseteq C, \mu \kappa_{A}$ is null on $B$ so that there exists $v: A / B \rightarrow S(A) / C$ such that $\mu \kappa_{A}=v \pi$

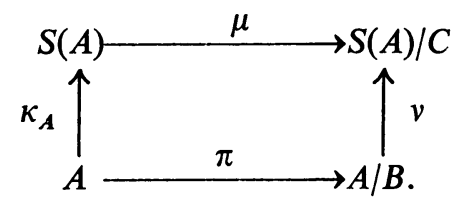

Then $\kappa_{S(A) / c} \mu \kappa_{A}=\kappa_{S(A) / c} v \pi=S(v) \kappa_{A / B} \pi=S(v) S(\pi) \kappa_{A}$ so that $\kappa_{S(A) / c} \mu=S(v) S(\pi)$. Since $S(A) / C$ is $\mathfrak{r}_{\mathfrak{B}}$-separated, $\kappa_{S(A) / C}$ is a monomorphism so that

$$
C=\operatorname{ker} \mu \supseteq \operatorname{ker} S(\pi)=\rho(B) .
$$

(2) $\kappa_{A}^{-1}(\rho(B))=\pi^{-1}\left(\mathfrak{r}_{\mathfrak{B}}(A / B)\right)$. This is immediate since, by definition, $\kappa_{A}^{-1}(\rho(B))$ is the kernel of $S(\pi) \kappa_{A}=\kappa_{A / B} \pi$.

(3) $\kappa_{A}^{-1}(\rho(B))$ is the largest of those $R$-submodules $C$ of $A$ for which $\rho(C)=\rho(B)$ $\left(\kappa_{A}^{-1}(\rho(B))\right.$ may be thought of as a generalized isolated component of $\left.B\right)$.

Proof. Let $\pi^{\prime}$ denote the natural homomorphism from $A$ onto $A / \kappa_{A}^{-1}(\rho(B))$. Then there exists a unique homomorphism $\gamma: A / B \rightarrow A / \kappa_{A}^{-1}(\rho(B))$ such that $\pi^{\prime}=\gamma \pi$. Obviously, $\gamma$ is an epimorphism, and by (2), the kernel of $\gamma$ is $\mathfrak{r}_{\mathfrak{B}}(A / B)$. Then $\gamma \in \subseteq$ and it is easy to verify that $S(\gamma): S(A / B) \rightarrow S\left(A / \kappa_{A}^{-1}(\rho(B))\right.$ must also be in $\subseteq$ so that, since $S(A / B) \in \mathfrak{Q}, S(\gamma)$ is a retraction and therefore a monomorphism (one could even show that it is an isomorphism). But since $S\left(\pi^{\prime}\right)=S(\gamma) S(\pi)$,

$$
\rho\left(\kappa_{A}^{-1}(\rho(B))\right)=\operatorname{ker} S\left(\pi^{\prime}\right)=\operatorname{ker} S(\pi)=\rho(B) .
$$

Now, if $C$ is an $R$-submodule of $A$ for which $\rho(C)=\rho(B)$ and if $\pi^{\prime \prime}$ denotes the natural homomorphism of $A$ onto $A / C$, then

$$
C \subseteq \pi^{\prime \prime-1}\left(\mathfrak{r}_{\mathfrak{F}}(A / C)\right)=\kappa_{A}^{-1}(\rho(C))=\kappa_{A}^{-1}(\rho(B)) .
$$

In $\S 5$, it will be shown by an example that $\rho(B)$ is in general not necessarily generated as an $S(R)$-module by $\kappa_{A}(B)$.

However, if the three equivalent statements of Theorem 2 hold, then for any module $A$ in $\mathscr{C}_{R}, S(A) \cong A \otimes_{R} S(R)$ is generated by $\kappa_{A}(A)$ as an $S(R)$-module and $S$ is right exact. Then if $B \subseteq A$ and if $f$ and $g$ denote the natural injection of $B$ in $A$ and the natural projection of $A$ onto $A / B$, respectively, we have a commutative diagram

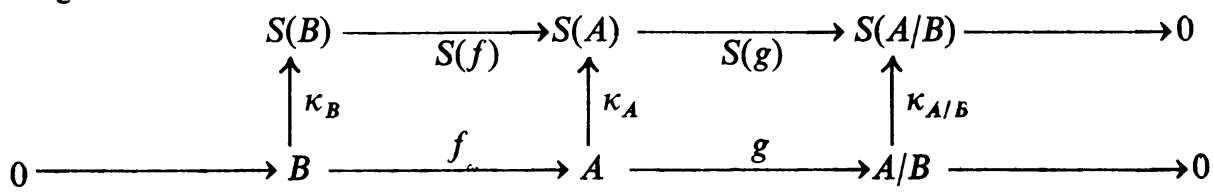


with exact rows. Since $S(B)$ is generated by $\kappa_{B}(B)$ as an $S(R)$-module, $\rho(B)=\operatorname{ker} S(g)=\operatorname{im} S(f)$ is generated as an $S(R)$-module by $S(f) \kappa_{B}(B)=\kappa_{A}(B)$.

It would not be difficult to extend the scope of this section by studying categories of $R$-modules with a type of structure compatible with their $R$-module structure and $R$-homomorphisms compatible with this type of structure.

A particular case of such an extended theory would be the following: choose a fixed topology of $R$, compatible with the operations, and in the category whose objects are the topological $R$-modules (the scalar product is a continuous function of two variables) and whose morphisms are the continuous $R$-homomorphisms, consider the regular injective structure whose injectives are the complete and Hausdorff topological $R$-modules.

6. Rational injective structures and rings of quotients. Let $A$ be a right $R$-module, let $B$ be an $R$-submodule of $A$, and let $C$ be a subset of $A$. We define $B: C=\{x \in R \mid C x \subseteq B\} . B: C$ is a right ideal of $R$.

Definition 1. A preradical $\mathfrak{r}$ of $\mathscr{C}_{R}$ will be said to be a torsion preradical if, for any module $A$ in $\mathscr{C}_{R}, B \subseteq \mathfrak{r}(A)$ implies that $\mathfrak{r}(B)=B$ or, equivalently, $B \subseteq A$ implies that $\mathfrak{r}(B)=\mathfrak{r}(A) \cap B$, or again, if $\mathfrak{r}$, considered as a functor of $\mathscr{C}_{R}$ into itself, is left exact.

We notice that the finest and coarsest preradicals of $\mathscr{C}_{R}$ are torsion preradicals.

Proposition 1. If $\mathfrak{r}$ is a torsion preradical of $\mathscr{C}_{R}$, then for any module $A$ of $\mathscr{C}_{R}$,

$$
\mathfrak{r}(A)=\left\{a \in A \mid(0: a) \leqq_{\mathfrak{r}} R\right\}
$$

Proof. Let $a \in A$. The kernel of $\phi_{a}$ is $(0: a)$ and the image of $\phi_{a}$ is $a R$. But it is obvious that $a \in \mathfrak{r}(A)$ iff $\mathfrak{r}(a R)=a R$, i.e., iff $(0: a) \leqq_{\mathfrak{r}} R$.

Proposition 2. If $\mathfrak{r}$ is a torsion preradical of $\mathscr{C}_{R}$, then the relation " $\leqq \mathfrak{r}$ " has the following properties:

F1. For all $A, A \leqq{ }_{\mathfrak{r}} A$.

F2. If $A \subseteq B \subseteq C$, there $A \leqq{ }_{\mathfrak{r}} C$ implies that $A \leqq{ }_{\mathfrak{r}} B$ and $B \leqq{ }_{\mathfrak{r}} C$.

F3. If $A \leqq{ }_{\mathfrak{r}} B$ and if $\phi$ is any homomorphism into $B$, then $\phi^{-1}(A) \leqq{ }_{\mathfrak{r}} \phi^{-1}(B)$.

F4. If $A \subseteq B$ and if for every $x \in B / A,(0: x) \leqq_{\mathfrak{r}} R$, then $A \leqq{ }_{\mathfrak{r}} B$.

If furthermore $\mathfrak{r}$ is a radical, then we have

F5. $A \leqq_{\mathfrak{r}} B$ and $B \leqq_{\mathfrak{r}} C$ imply that $A \leqq{ }_{\mathfrak{r}} C$.

Proof. F1 is obvious and the second half of F2 has already been established (Proposition 2, §3). Assume that $A \subseteq B \subseteq C$ and that $A \leqq{ }_{\mathfrak{r}} C$. Then, $\mathfrak{r}(C / A)=$ $C / A \supseteq B / A$ so that $\mathfrak{r}(B / A)=B / A$, i.e., $A \leqq{ }_{\mathfrak{r}} B$.

Now assume that $A \leqq_{\mathrm{r}} B$ and that $\phi$ is a homomorphism into $B$. Then $\phi^{-1}(B) / \phi^{-1}(A) \cong \operatorname{im} \phi /(\operatorname{im} \phi \cap A) \cong(\operatorname{im} \phi+A) / A \subseteq B / A=\mathfrak{r}(B / A)$ so that $\mathfrak{r}\left(\phi^{-1}(B) / \phi^{-1}(A)\right)=\phi^{-1}(B) / \phi^{-1}(A)$, i.e., $\phi^{-1}(A) \leqq \mathfrak{r} \phi^{-1}(B)$.

F4 is immediate by the definition of " $\leqq$ " " and the preceding proposition.

That F5 holds when $\mathfrak{r}$ is a radical was seen in Proposition 2 of $\S 3$. 
COROLLARY 1. If $\mathfrak{r}$ is a torsion preradical of $\mathscr{C}_{R}$,

$$
A \leqq{ }_{\mathfrak{r}} B \& C \subseteq B \Rightarrow A \cap C \leqq{ }_{\mathfrak{r}} C .
$$

COROLLARY 2. If $\mathfrak{r}$ is a torsion radical of $\mathscr{C}_{R}$,

$$
A \leqq{ }_{\mathfrak{r}} B \& C \leqq{ }_{\mathfrak{r}} B \Rightarrow A \cap C \leqq{ }_{\mathfrak{r}} B .
$$

Proof. By Corollary $1, A \cap C \leqq_{\mathfrak{r}} C$, and by F5, $A \cap C \leqq_{\mathfrak{r}} B$.

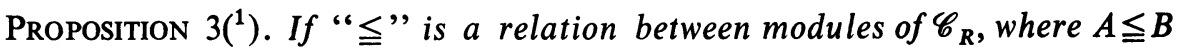
implies $A \subseteq B$, which satisfies $\mathrm{F} 1$ to $\mathrm{F} 4$, then the set $\mathscr{S}$ of all right ideals $A$ of $R$ such that $A \leqq R$ has the following properties:

G1. $A \in \mathscr{S}$ and $A \subseteq B \subseteq R$ imply that $B \in \mathscr{S}$.

G2. $A \in \mathscr{S}$ and $B \in \mathscr{S}$ imply that $A \cap B \in \mathscr{S}$.

G3. If $A \in \mathscr{S}$, then for any $x \in R,(A: x) \in \mathscr{S}$.

If furthermore, the relation " $\leqq$ " has the property $\mathrm{F} 5$, then $\mathscr{S}$ has the property G4. If $A \subseteq B$, if $B \in \mathscr{S}$, and if for each $x \in B,(A: x) \in \mathscr{S}$, then $A \in \mathscr{S}$.

Proof. That G1 and G2 hold follows from F2 and Corollary 2 of the preceding proposition.

If $A \in \mathscr{S}$ and $x \in R$, then $A: x=\phi_{x}^{-1}(A)$ so that by $\mathrm{F} 3,(A: x) \leqq R$ and $\mathrm{G} 3$ is established. If the relation " $\leqq$ " satisfies $F 5$, under the hypotheses of $G 4$, if $\phi$ denotes the natural homomorphism of $R$ onto $R / A$, then $A: x$ is the kernel of $\phi \phi_{x}$ which maps 1 onto $\phi(x)$, i.e., $A: x=0: \phi(x)$, so that $A \leqq B$ by F4. Therefore, by F5, $A \leqq R$, i.e., $A \in \mathscr{S}$.

Corollary. If $\mathrm{G} 4$ is satisfied, then $A \in \mathscr{S} \& B \in \mathscr{S} \Rightarrow A B \in \mathscr{S}$.

Proof. If $x \in A$, then $x B \subseteq A B$ so that $B \subseteq A B: x$. By G1, $(A B: x) \in \mathscr{S}$. Therefore, by $\mathrm{G} 4, A B \in \mathscr{S}$.

We notice that if for every $A \in \mathscr{S}$ there exists a two-sided ideal $B \in \mathscr{S}$ contained in $A$, in particular, if $R$ is commutative, then $A: x \supseteq B: x \supseteq B \in \mathscr{S}$ so that, by $\mathrm{G} 1, A: x \in \mathscr{S}$, i.e., $\mathrm{G} 3$ is a consequence of $\mathrm{G} 1$.

PROPOSITION 4. If $\mathscr{S}$ is a set of right ideals of $R$ satisfying conditions $\mathrm{G} 1$ to $\mathrm{G} 3$, then there exists one and only one torsion preradical $\mathfrak{x}$ of $\mathscr{C}_{R}$ for which $\mathscr{S}$ is the set of all right ideals $A$ of $R$ such that $A \leqq{ }_{\mathrm{r}} R$. If, furthermore, $\mathscr{S}$ satisfies $\mathrm{G} 4$, then $\mathfrak{r}$ is a radical.

Proof. The uniqueness of $\mathfrak{r}$ follows from Proposition 1. For each module $A$ in $\mathscr{C}_{R}$, set

$$
\mathfrak{r}(A)=\{a \in A \mid(0: a) \in \mathscr{S}\} .
$$

If $a, b \in \mathfrak{r}(A)$, since $(0: a+b) \supseteq(0: a) \cap(0: b)$, by G1 and G2, $(0: a+b) \in \mathscr{S}$

(1) Added in proof. These sets of right ideals and the rational injective structures they define have also been considered by P. Gabriel (Bull. Soc. Math. France 90 (1963), no. 3). 
so that $a+b \in \mathfrak{r}(A)$. If $a \in \mathfrak{r}(A)$ and if $x \in R$, then since $0: a x=(0: a): x$, by G3, $0: a x \in \mathscr{S}$, i.e., $a x \in \mathfrak{r}(A)$. Therefore $\mathfrak{r}(A)$ is an $R$-submodule of $A$. If $f: A \rightarrow B$, since for any $a \in A, 0: f(a) \supseteq 0: a$, by $\mathrm{G} 1, f$ maps $\mathfrak{r}(A)$ into $\mathfrak{r}(B)$. We have thus established that $\mathfrak{r}$ is a preradical of $\mathscr{C}_{R}$. That it is a torsion preradical is immediate from its definition.

Now assume that $\mathscr{S}$ satisfies G4. Let $A$ be any module in $\mathscr{C}_{R}$ and let $\phi$ be the natural homomorphism of $A$ onto $A / \mathfrak{r}(A)$. If $x \in \mathfrak{r}(A / \mathfrak{r}(A))$, pick $y \in A$ such that $\phi(y)=x$. Then $\phi \phi_{y}$ maps 1 onto $x$ so that $\phi_{y}^{-1}(\mathfrak{r}(A))=\operatorname{ker} \phi \phi_{y}=0: x \in \mathscr{S}$. Then, for each $z \in \phi_{y}^{-1}(\mathfrak{r}(A))$, since $(0: y): z=0: y z=0: \phi_{y}(z) \in \mathscr{S}$, by G4, $(0: y) \in \mathscr{S}$ so that $y \in \mathfrak{r}(A)$ and therefore $x=0$. Therefore $\mathfrak{r}$ is a radical of $\mathscr{C}_{R}$.

From now on, given a torsion preradical $\mathfrak{r}$ of $\mathscr{C}_{R}$, we will denote the set of all $\mathfrak{r}$-dense right ideals of $R$ by $\mathscr{S}_{\mathfrak{r}}$, we will denote by $\tilde{\mathfrak{Q}}_{\mathfrak{r}}$ the class of all modules of $\mathscr{C}_{R}$ that are injectives with respect to the natural injections of ideals in $\mathscr{S}_{\mathfrak{r}}$ in $R$, and we will denote by $\mathfrak{Q}_{\mathfrak{r}}$ the class of all $\mathfrak{r}$-separated modules in $\tilde{\mathfrak{Q}}_{\mathfrak{r}}$.

LEMMA 1. If $\mathfrak{r}$ is a torsion radical of $\mathscr{C}_{R}$, and if $A$ is any module in $\mathscr{C}_{R}$, then there exists an extension $A$ of $A$ such that

(1) $A \leqq{ }_{\mathfrak{r}} \bar{A}$.

(2) $\bar{A}$ is an essential extension of $A$.

(3) If $f: C \rightarrow A$ where $C \in \mathscr{S}_{\mathfrak{r}}$, then $f$ may be extended to $g: R \rightarrow \bar{A}$.

Furthermore, if $\mathfrak{r}(A)=0$, then $\mathfrak{r}(\bar{A})=0$ and $\bar{A} \in \mathfrak{Q}_{\mathfrak{r}}$.

Proof. Let $\left\{f_{i}\right\}_{i \in I}$ be the family of all homomorphisms from ideals in $\mathscr{S}_{\mathfrak{r}}$ into $A$ and let $D$ be the direct sum of $\left\{R_{i}\right\}_{i \in I}$, where for each $i \in I, R_{i} \cong R$. For each $i \in I, \operatorname{dom} f_{i}$ is a submodule of $R_{i}$; the sum $E$ of these submodules in $D$ is direct and, obviously, $D / E$ is the direct sum of the family $\left\{R_{i} / \operatorname{dom} f_{i}\right\}_{i \in I}$ so that $\mathfrak{r}(D / E)=(D / E)$. There exists $f: E \rightarrow A$ such that for each $i \in I, f_{i}$ is the restriction of $f$ to $\operatorname{dom} f_{i}$. Let $g$ denote the injection of $E$ into $D$ and let $(B, \alpha, \beta)$ be a direct sum of $A$ and $D$ with $f$ and $g$ amalgamated. We then have the commutative diagram with exact rows

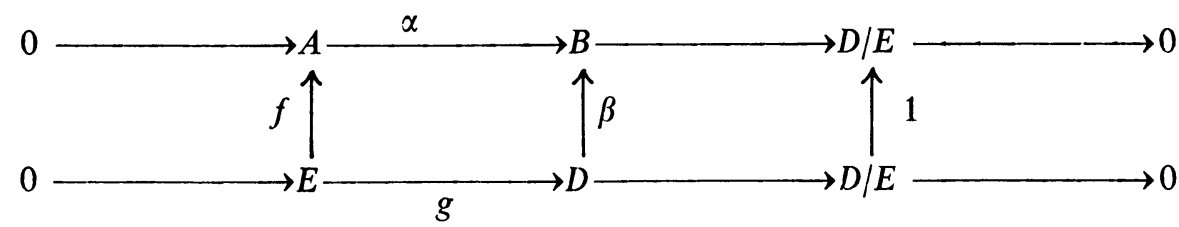

so that $\alpha$ is a monomorphism and $\alpha(A) \leqq_{\mathfrak{r}} B$. We may think of $B$ as a true extension of $A$. It is then obvious that the restriction of $\beta$ to $R_{i}$ is an extension of $f_{i}$. Now, if $G$ is a submodule of $B$ which is maximal with respect to the property $G \cap A=0$, then the natural homomorphism $\phi$ of $B$ onto $B / G=\bar{A}$ induces an isomorphism on $A$ so that $\bar{A}$ may be considered as a true extension of $A$ and $A$ is an essential extension of $A$. That it also has the other two required properties is obvious. 
Now, assume that $\mathfrak{r}(A)=0$. Since $\mathfrak{r}$ is a torsion radical

$$
0=\mathfrak{r}(A)=\mathfrak{r}(\bar{A}) \cap A,
$$

and since $\bar{A}$ is an essential extension of $A, \mathfrak{r}(\bar{A})=0$. Then, let $f: C \rightarrow \bar{A}$ where $C \in \mathscr{S}_{\mathfrak{r}}$ and let $g$ be the restriction of $f$ to $f^{-1}(A)$. By F3, $f^{-1}(A) \leqq_{\mathrm{r}} C$ so that by F2, $f^{-1}(A) \in \mathscr{S}_{\mathfrak{r}}$. Therefore there exists $h: R \rightarrow A$ extending $g$ and since $f^{-1}(A) \leqq{ }_{\mathrm{r}} C$ and $\bar{A}$ is separated, by Proposition 4 of $\S 3, h$ must coincide with $f$ on $C$. Therefore, $\bar{A}$ in this case is in $\mathfrak{Q}_{\mathfrak{r}}$.

Lemma 2. If $\mathfrak{r}$ is a torsion radical of $\mathscr{C}_{R}$, then for any module $A$ of $\mathscr{C}_{R}$, there exists an extension $Q$ of $A$ such that

(1) $A \leqq_{\mathfrak{r}} Q$.

(2) $Q$ is an essential extension of $A$.

(3) $Q \in \tilde{\mathbb{Q}}_{\mathbf{r}}$.

Proof. In the case where $\mathfrak{r}(A)=0$, Lemma 1 already gives us a solution. However, in general, one must apply Lemma 1 a transfinite number of times. We define a transfinite sequence of extensions of $A$ as follows: $A_{0}=A$ and if for each $\beta<\alpha, A_{\beta}$ has been defined, then if $\alpha$ is not a limit ordinal we set $A_{\alpha}=\bar{A}_{\alpha-1}$ while if $\alpha$ is a limit ordinal, we set $A_{\alpha}=\bigcup_{\beta<\alpha} A_{\beta}$. It is easy to verify that for each $\alpha, A \leqq_{\mathfrak{r}} A_{\alpha}$ and $A_{\alpha}$ is an essential extension of $A$. Now let $\gamma$ be the least infinite ordinal whose cardinal is larger than the cardinal of $R$, let $Q=A_{\gamma}$ and let $f: C \rightarrow Q$ where $C \in \mathscr{S}_{\mathbf{r}}$. By the choice of $\gamma$, there exists $\alpha<\gamma$ such that $f(C) \subseteq A_{\alpha}$. Then there exists $g: R \rightarrow A_{\alpha+1} \subseteq Q$ extending $f$, so that $Q \in \tilde{Q}_{\mathfrak{r}}$.

THEOREM 1. If $\mathfrak{r}$ is a torsion radical of $\mathscr{C}_{R}$, then $\tilde{\mathfrak{Q}}_{\mathfrak{r}}$ is the class of injectives of an injective structure $\tilde{\mathfrak{B}}_{\mathfrak{r}}\left(\tilde{\mathfrak{S}}_{\mathfrak{r}}, \tilde{\mathfrak{Q}}_{\mathfrak{r}}\right)$, where $\tilde{\mathfrak{S}}_{\mathfrak{r}}$ is the class of all monomorphisms $f: A \rightarrow B$ for which there exists an epimorphism $g: B \rightarrow C$ such that $g f$ is a monomorphism and $g f(A) \leqq{ }_{\mathrm{r}} C$.

Proof. That $\Psi \Phi\left(\tilde{Q}_{\mathfrak{r}}\right)=\tilde{Q}_{\mathfrak{r}}$ is obvious. To show that every $Q \in \tilde{Q}_{\mathfrak{r}}$ is injective with respect to every homomorphism in $\tilde{\mathfrak{S}}_{r}$ it is obviously sufficient to show that such a module $Q$ is injective with respect to any inclusion $A \varliminf_{\mathrm{r}} C$. Let $f: A \rightarrow Q$ and let $g$ be a maximal extension of $f$ to a homomorphism from a submodule of $C$ into $Q$. For any $x \in C$, since $\mathfrak{r}(C / A)=C / A, A: x \in \mathscr{S}_{\mathfrak{r}}$ and since dom $g: x \supseteq A: x$, $\operatorname{dom} g: x \in \mathscr{S}_{\mathfrak{r}}$. Then if $\psi$ denotes the restriction of $\phi_{x}$ to dom $g: x, \psi$ maps dom $g: x$ into dom $g$ and $g \psi$ maps dom $g: x$ into $Q$. Since $Q \in \tilde{\mathfrak{Q}}_{\mathfrak{r}}, g \psi$ may be extended to $\chi: R \rightarrow Q$. Now if for each $x r+y \in x R+\operatorname{dom} g$, one sets

$$
\zeta(x r+y)=u r+g(y)
$$

where $u=\chi(1)$, it is easy to show that this defines an $R$-homomorphism $\zeta:(x R+\operatorname{dom} g) \rightarrow Q$ extending $g$, so that by the choice of $g, x \in \operatorname{dom} g$. Therefore $\operatorname{dom} g=C$. 
If $A$ is any module of $\mathscr{C}_{R}$, then Lemma 2 shows that there exists a homomorphism $\phi: A \rightarrow Q$ in $\tilde{\Xi}_{\mathfrak{r}}$ where $Q \in \tilde{Q}_{\mathfrak{r}}$. Finally if $f: A \rightarrow B$ is a homomorphism with respect to which all modules in $\tilde{\mathfrak{Q}}_{\mathfrak{r}}$ are injective, there exists $g: B \rightarrow Q$ such that $\phi=g f$ so that $f$ is a monomorphism and if we consider $g$ as a map from $B$ onto $f(B)=C, g f$ is a monomorphism, and $g f(A) \leqq{ }_{\mathfrak{r}} C$.

Corollary 1. If $A$ is any module of $\mathscr{S}_{R}$ and if $Q$ is an extension of $A$ whose existence is asserted in Lemma 2, then $Q$ is maximal with respect to the properties (1) $A \leqq{ }_{\mathfrak{r}} Q$ and (2) $Q$ is an essential extension of $A$.

Corollary 2. A module $A$ of $\mathscr{C}_{R}$ is in $\tilde{\mathfrak{Q}}_{\mathfrak{r}}$ if and only if it has no properessential extension $B$ such that $A \leqq{ }_{\mathfrak{r}} B$.

CoRollary 3. If $A$ is any module of $\mathscr{C}_{R}$ and if $Q$ and $Q^{\prime}$ are two extensions of $A$ with the properties given in Lemma 2, then there exists an isomorphism of $Q$ onto $Q^{\prime}$ which induces the identity on $A$.

The reader will recognize in the proofs of Lemmas 1 and 2 and of Theorem 1 arguments due to R. Baer [1].

If $A$ is any $\mathfrak{r}$-separated module of $\mathscr{C}_{R}$, we will now give another method for constructing the extension $A$ of $A$ whose existence is asserted in Lemma 1. (It is based on the method of $\mathrm{R}$. E. Johnson for constructing the extended centralizer of a ring over a module [8].)

We will call the homomorphisms from right ideals in $\mathscr{S}_{\mathfrak{r}}$ into $A$ the $\mathfrak{r}$-fractional homomorphisms from $R$ to $A$.

LEMMA 3. If $f$ is an $\mathfrak{r}$-fractional homomorphism from $R$ to $A$ then $f$ possesses a unique maximal extension which is, of course, also an $\mathfrak{r}$-fractional homomorphism from $R$ to $A$.

Proof. Let $f^{\prime}$ and $f^{\prime \prime}$ be two maximal extensions of $f$ and let $\bar{f}^{\prime}$ and $\bar{f}^{\prime \prime}$ denote their restrictions to $\operatorname{dom} f^{\prime} \cap \operatorname{dom} f^{\prime \prime}$. Since $\operatorname{dom} f \leqq_{\mathfrak{r}} R, \operatorname{dom} f \leqq_{\mathfrak{r}} \operatorname{dom} f^{\prime} \cap \operatorname{dom} f^{\prime \prime}$. Then, since $A$ is $\mathfrak{r}$-separated, $\bar{f}^{\prime}=\bar{f}^{\prime \prime}$. Then if for each $x^{\prime} \in \operatorname{dom} f^{\prime}$ and each $x^{\prime \prime} \in \operatorname{dom} f^{\prime \prime}$ one sets $g\left(x^{\prime}+x^{\prime \prime}\right)=f^{\prime}\left(x^{\prime}\right)+f^{\prime \prime}\left(x^{\prime \prime}\right)$ it is easy to verify that this defines an $R$-homomorphism $g: \operatorname{dom} f^{\prime}+\operatorname{dom} f^{\prime \prime} \rightarrow A$ extending both $f^{\prime}$ and $f^{\prime \prime}$ and since $f^{\prime}$ and $f^{\prime \prime}$ are both maximal, $f^{\prime}=f^{\prime \prime}$.

We will denote the set of all maximal $\mathfrak{r}$-fractional homomorphisms from $R$ to $A$ by $\operatorname{Fr}_{\mathfrak{r}}(R, A)$. We turn $\operatorname{Fr}_{\mathfrak{r}}(R, A)$ into a right $R$-module as follows:

If $f, g \in \operatorname{Fr}_{\mathfrak{r}}(R, A)$ and if $\bar{f}$ and $\bar{g}$ denote the restrictions of $f$ and $g$ to $\operatorname{dom} f \cap \operatorname{dom} g \leqq_{\mathrm{r}} R$, by Lemma $3, \bar{f}+\bar{g}$ possesses a unique maximal extension which is in $\operatorname{Fr}_{\mathfrak{r}}(R, A)$ and which we define to be $f+g$. Then if $f, g, h \in \operatorname{Fr}_{\mathfrak{r}}(R, A)$ and if $f, g$ and $h$ denote restrictions of $f, g$ and $h$ to $\operatorname{dom} f \cap \operatorname{dom} g \cap \operatorname{dom} h \leqq{ }_{\mathfrak{r}} R$, $(f+g)+h$ and $f+(g+h)$ are both equal to the unique maximal extension of $(\bar{f}+\bar{g})+h=\bar{f}+(\bar{g}+h)$. The 0 -map from $R$ to $A$ is obviously a unity element for this addition and if $f \in \operatorname{Fr}_{\mathfrak{r}}(R, A),-f: \operatorname{dom} f \rightarrow A$ is obviously the additive 
inverse of $f$ in $\operatorname{Fr}_{\mathfrak{r}}(R, A)$. Therefore $\operatorname{Fr}_{\mathfrak{r}}(R, A)$ is an abelian group under this operation, obviously containing $\operatorname{Hom}_{R}(R, A) \cong A$.

Now if $r \in R$ and $f \in \operatorname{Fr}_{\mathfrak{r}}(R, M)$ and if for each $x \in \operatorname{dom} f: r$ we set $f_{r}(x)=f(r x)$, then $f_{r}$ is an $R$-homomorphism from $(\operatorname{dom} f: r) \in \mathscr{S}_{\mathfrak{r}}$ into $A$ since

$$
\begin{aligned}
f_{r}(x+y) & =f(r(x+y))=f(r x+r y)=f(r x)+f(r y)=f_{r}(x)+f_{r}(y), \\
f_{r}\left(x r^{\prime}\right) & =f\left(r\left(x r^{\prime}\right)\right)=f\left((r x) r^{\prime}\right)=(f(r x)) r^{\prime}=\left(f_{r}(x)\right) r^{\prime} .
\end{aligned}
$$

Then, $f_{r}$ possesses a maximal extension in $\operatorname{Fr}_{\mathfrak{r}}(R, A)$ which we define to be $f r$.

To show that $(f+g) r=f r+g r$, it suffices to show that these two fractional homomorphisms coincide on

$$
(\operatorname{dom} f \cap \operatorname{dom} g): r=(\operatorname{dom} f: r) \cap(\operatorname{dom} g: r) \in \mathscr{S}_{r} .
$$

But for any $x$ in this ideal,

$$
\begin{aligned}
((f+g) r)(x) & =(f+g)_{r}(x)=(f+g)(r x)=f(r x)+g(r x) \\
& =(f r)(x)+(g r)(x)=(f r+g r)(x) .
\end{aligned}
$$

To show that $f(r+s)=f r+f s$, it suffices to show that these two maps coincide on $(\operatorname{dom} f: r) \cap(\operatorname{dom} f: s)=\operatorname{dom} f:(r+s) \in \mathscr{S}_{\mathfrak{r}}$. But for any $x$ in this ideal,

$$
\begin{aligned}
(f(r+s))(x) & =f((r+s) x)=f(r x+s x)=f(r x)+f(s x) \\
& =(f r)(x)+(f s)(x)=(f r+f s)(x) .
\end{aligned}
$$

To show that $f(r s)=(f r) s$, it suffices to show that these two maps coincide on $(\operatorname{dom} f: r s)=(\operatorname{dom} f: r): s \in \mathscr{S}_{r}$. But for any $x$ in this right ideal

$$
(f(r s))(x)=f((r s) x)=f(r(s x))=(f r)(s x)=((f r) s)(x) .
$$

It is obvious that $f \cdot 1=f$ for any $f \in \operatorname{Fr}_{\mathfrak{r}}(R, A)$.

Thus, $\operatorname{Fr}_{\mathfrak{r}}(R, A)$ is a right $R$-module obviously containing the right $R$-module $\operatorname{Hom}_{R}(R, A)$ which is isomorphic to $A$. If $f \in \operatorname{Fr}_{\mathfrak{r}}(R, A)$ and if $r \in \operatorname{dom} f$, then $\operatorname{dom} f: r=R$ and since $\operatorname{dom} f r \supseteq \operatorname{dom} f: r, f r \in \operatorname{Hom}_{R}(R, A)$. Therefore, $\operatorname{Hom}_{R}(R, A): f \supseteq \operatorname{dom} f$ so that $\operatorname{Hom}_{R}(R, A): f \in \mathscr{S}_{\mathfrak{r}}$. Therefore, $\operatorname{Hom}_{R}(R, A) \leqq_{\mathfrak{r}} \operatorname{Fr}_{\mathfrak{r}}(R, A)$. Now assume that $f R \cap \operatorname{Hom}_{R}(R, A)=0$. If $r \in \operatorname{dom} f$, i.e., if $1 \in \operatorname{dom} f: r$, then as we have just seen above, $f r \in \operatorname{Hom}_{R}(R, A)$ so that $0=f r(1)=f_{r}(1)=f(r 1)=f(r)$ and therefore $f=0$. Therefore $\operatorname{Fr}_{r}(R, A)$ is an essential extension of $\operatorname{Hom}_{R}(R, A)$. If $f \in \operatorname{Fr}_{\mathfrak{r}}(R, A)$ and if $g$ is the homomorphism from $R$ to $\operatorname{Hom}_{R}(R, A)$ that maps 1 onto $f$, for each $r \in \operatorname{dom} f$,

$$
g(r)=f r \in \operatorname{Hom}_{R}(R, A)
$$

and $f r$ is to be identified with $f(r)$ by the canonical isomorphism between $\operatorname{Hom}_{R}(R, A)$ and $A$. Therefore $g$ may be considered as an extension of $f$.

We have thus shown that the extension $\operatorname{Fr}_{\mathfrak{r}}(R, A)$ of $\operatorname{Hom}_{R}(R, A) \cong A$ satisfies 
the conditions of Lemma 1 . However, it appears that Lemma 1 gives a simpler and more direct method for constructing such an extension of $A$. The reason we have given this other method here is that it permits one to compare our results with other results in the literature $[8 ; 11 ; 5]$.

THEOREM 2. If $\mathfrak{r}$ is a torsion radical of $\mathscr{C}_{R}$, then $\mathfrak{Q}_{\mathfrak{r}}$ is the class of injectives of the coarsest regular injective structure $\mathfrak{B}_{\mathfrak{r}}\left(\mathfrak{S}_{\mathfrak{r}}, \mathfrak{Q}_{\mathfrak{r}}\right)$ of $\mathscr{C}_{R}$ defining the radical $\mathfrak{r}$.

Proof. If $A$ is any module in $\mathscr{C}_{R}$, let $\phi$ denote the natural homomorphism of $A$ onto $A / \mathfrak{r}(A)$ and let $\psi$ denote the injection of $A / \mathfrak{r}(A)$ into $(\overline{A / \mathfrak{r}(A)}) \in \mathfrak{Q}_{\mathfrak{r}}$ (defined in Lemma 1). If $f: A \rightarrow Q$, where $Q \in \mathbb{Q}_{\mathfrak{r}}$, since $Q$ is $\mathfrak{r}$-separated, $\operatorname{ker} f \supseteq \mathfrak{r}(A)$ so that there exists a unique $g: A / \mathfrak{r}(A) \rightarrow Q$ such that $f=g \phi$. Since $\psi \in \tilde{\Xi}_{\mathfrak{r}}$ and $Q \in \tilde{\mathfrak{Q}}_{\mathfrak{r}}$, there exists $h: \overline{(A / \mathfrak{r}(A)} \rightarrow Q$ such that $g=h \psi$ so that $f=h \psi \phi$. If $f=h^{\prime} \psi \phi$, since $\operatorname{im} \psi \phi \leqq \widehat{r} \overline{(A / r(A))}$ and $Q$ is $\mathfrak{r}$-separated, by Proposition 4 of $\S 3, h=h^{\prime}$. By Proposition 4 of $\S 1, \mathfrak{Q}_{\mathfrak{r}}$ is the class of injectives of a regular injective structure $\mathfrak{B}_{\mathfrak{r}}\left(\mathfrak{S}_{\mathfrak{r}}, \mathfrak{Q}_{\mathfrak{r}}\right)$ obviously defining the radical $\mathfrak{r}$. Now let $\mathfrak{B}(\mathfrak{S}, \mathfrak{Q})$ be a regular injective structure of $\mathscr{C}_{R}$ defining the radical $\mathfrak{r}$ and let $A \in \mathfrak{Q}_{\mathfrak{r}}$. There exists $\phi: A \rightarrow Q$ in $\mathfrak{S}$, where $Q \in \mathfrak{Q}$ and since $\mathfrak{B}$ is regular, one may choose $\phi$ in such a way that for any $f: A \rightarrow Q^{\prime}$, where $Q^{\prime} \in \mathbb{Q}$, there exists a unique $g: Q \rightarrow Q^{\prime}$ such that $f=g \phi$. By Proposition 4 of $\S 3, \operatorname{im} \phi \leqq{ }_{\mathfrak{r}} Q$. Since $A$ is $\mathfrak{r}$-separated, $\phi$ is a monomorphism so that $\phi \in \tilde{\mathcal{G}}_{\mathfrak{r}}$. Then $\phi$ is a retraction so that $A \in \mathfrak{Q}$. Therefore, $\mathfrak{V}_{\mathfrak{r}}$ is coarser than $\mathfrak{B}$.

We will call $\mathfrak{P}_{\mathfrak{r}}$ the rational injective structure determined by $\mathfrak{r}$. If $\kappa: 1_{\mathscr{C}_{R}} \rightarrow S$ is a regular functorial description of $\mathfrak{B}_{\mathfrak{r}}$, then for any module $A$, we will call $S(A)$ the $\mathfrak{r}$-rational completion of $A$ and, as we saw in $\S 5, S(R)$ may be turned into a ring which we will call the ring of quotients of $R$ determined by $\mathfrak{r}$.

THEOREM 3. If $\mathfrak{r}$ is a torsion radical of $\mathscr{C}_{R}$ and if $\kappa: 1_{\mathscr{C}_{R}} \rightarrow S$ is a regular description of $\mathfrak{B}_{\mathfrak{r}}$, then given any ring homomorphism $\psi: R \rightarrow R^{\prime}$, if $R^{\prime}$, turned into a right $R$-module in the natural way, is $\mathfrak{r}$-separated and has the property that any right $R$-homomorphism from some right ideal $A \in \mathscr{S}_{\mathfrak{r}}$ to $\psi(R)$ may be extended to a right $R$-homomorphism from $R$ to $R^{\prime}$, then there exists a unique ring homomorphism $\chi: S(R) \rightarrow R^{\prime}$ such that $\psi=\chi \kappa_{R}$.

Proof. Let $\phi$ denote the natural homomorphism from $R^{\prime}$ onto $R^{\prime} / \psi(R)$ and let $C=\phi^{-1}\left(\mathfrak{r}\left(R^{\prime} / \psi(R)\right)\right)$. Then any right $R$-homomorphism $f: A \rightarrow \psi(R)$, where $A \in \mathscr{S}_{\mathfrak{r}}$, may be extended to a right $R$-homomorphism $g: R \rightarrow R^{\prime}$ and since $A \leqq{ }_{\mathrm{r}} R$, $g$ maps $R$ into $C$. If $D$ is a submodule of $C$ such that $D \cap \psi(R)=0$, then obviously, $\mathfrak{r}(D)=D$, and since $R^{\prime}$ is $\mathfrak{r}$-separated, $D=0$. Therefore $C=\overline{\psi(R)}$ (see Lemma 1 ) and since $C$ is $\mathfrak{r}$-separated, $C \in \mathfrak{Q}_{\mathfrak{r}}$. Our theorem then follows immediately from Theorem 3 of $\S 5$.

EXAMPLE 1. $\mathfrak{r}$ is the largest radical of $\mathscr{C}_{R}$. In this case, $A \leqq_{\mathfrak{r}} B$ just means that $A \subseteq B$ so that $\tilde{\mathfrak{G}}_{\mathfrak{r}}$ is just the class of all monomorphisms and $\tilde{\mathfrak{Q}}_{\mathfrak{r}}$ is just the class of all ordinary injective modules, i.e., $\tilde{\mathfrak{V}}_{\mathfrak{r}}$ is the exact injective structure of $\mathscr{C}_{R}$. 
Since 0 is the only $\mathfrak{r}$-separated module, $\mathfrak{B}_{\mathfrak{r}}$ is just the coarsest injective structure of $\mathscr{C}_{R}$.

EXAMPLE 2. $\mathfrak{r}$ is the smallest radical of $\mathscr{C}_{R}$. In this case, $\tilde{\mathfrak{B}}_{\mathfrak{r}}$ and $\mathfrak{B}_{\mathfrak{r}}$ are both equal to the finest injective structure of $\mathscr{C}_{R}$.

EXAmple 3. We define $A \leqq B$ to mean $A \leqq B(M)$ in the sense of [5] where $M$ is an arbitrary but fixed module in $\mathscr{C}_{R}$, i.e., any partial homomorphism $\phi$ from $B$ to $M$ for which $A \subseteq \operatorname{ker} \phi$ must be 0 . As was shown in [5], this relation satisfies F1, F2, F3 and F5. We show that it also satisfies F4. Assume that $A \subseteq B$ and that for each $x \in B / A,(0: x) \leqq R$. Let $\phi$ be a partial homomorphism from $B$ to $M$ such that $A \subseteq \operatorname{ker} \phi$. If $b$ is in the domain of definition of $\phi$, then $\phi \phi_{b}$ maps 1 onto $\phi(b)$ so that $\operatorname{ker} \phi \phi_{b}=0: \phi(b) \leqq R$ and therefore $\phi \phi_{b}=0$. Then, $\phi(b)=\phi \phi_{b}(1)=0$, so that $\phi=0$.

With the radical $\mathfrak{r}$ defined in this way, we see that $M$ is $\mathfrak{r}$-separated and that $\mathfrak{r}$ is the largest torsion radical with respect to which $M$ is $\mathfrak{r}$-separated. If $M=R$, then we denote the torsion radical defined in this way and the corresponding ring of quotients by by $\mathfrak{r}_{1}$ and $R_{1}$, respectively; $R_{1}$ is the ring of quotients of $R$ studied in $[8 ; 11 ; 5]$. However, the rational completion of a module studied in [5] is in general not the $\mathfrak{r}_{1}$-rational completion of this module. Note that, in general, given a torsion radical $\mathfrak{r}, M$ is $\mathfrak{r}$-separated if and only if $A \leqq R(M)$ for each $A \in \mathscr{S}_{\mathfrak{r}}$.

Consider the case where $R$ is the ring of all countably infinite diagonal matrices with entries in a field $F$, where all but a finite number of diagonal entries are equal. As was shown in [5], the subset $N$ of all matrices in $R$ such that all their entries, except for a finite number, are null is an ideal of $R$ with the property $N \leqq R(R)$ and $R_{1}$ is the ring of all countably infinite diagonal matrices with entries in $F$. Obviously, $\rho(N)=R_{1}$ (the correspondence $\rho$ was defined at the end of $\S 5)$. However, $N$ is obviously an ideal of $R_{1}$ so that $N R_{1}=N \neq R_{1}=\rho(N)$. Thus, if $(S, \kappa)$ is a regular description of the injective structure studied, the functors $S$ and $\mathscr{C}_{R} \otimes_{R} R_{1}$ are not equivalent.

EXAMPLE 4. Let $\mathscr{E}$ be a multiplicatively closed set of (two-sided) ideals of $R$, each of which is finitely generated as a right $R$-module. Then. the set $\mathscr{S}$ of all right ideals of $R$ that contain some ideal in $\mathscr{E}$ obviously satisfies G1 to G3. We show that it also satisfies G4. If $A \subseteq B$ and $B \in \mathscr{S}$, and if for each $x \in B$, $(A: x) \in \mathscr{S}$, then given any $y \in R$, since $B \in \mathscr{S}$, there exists $C \in \mathscr{E}$ such that $y C \subseteq B$. But $C=x_{1} R+\cdots+x_{n} R$. Since $y x_{i} \in B,\left(A: y x_{i}\right) \in \mathscr{S}$ so that there exists $C_{i} \in \mathscr{E}$ such that $y x_{i} C_{i} \subseteq A$. Then,

$$
\begin{aligned}
y C C_{1} C_{2} \cdots C_{n} & =y\left(x_{1} R+\cdots+x_{n} R\right) C_{1} C_{2} \cdots C_{n} \\
& \subseteq y x_{1} C_{1}+\cdots+y x_{n} C_{n} \subseteq A .
\end{aligned}
$$

Since $C C_{1} C_{2} \cdots C_{n} \in \mathscr{E},(A: y) \in \mathscr{S}$. Therefore, $A \in \mathscr{S}$.

LEMMA 4. If $\mathscr{E}$ is a multiplicatively closed set of ideals of $R$, each of which is finitely generated as a right $R$-module, if $\mathfrak{r}$ denotes the torsion radical defined 
by $\mathscr{E}$ and if $M$ is an $\mathfrak{r}$-separated module, then $M \in \mathfrak{Q}_{\mathfrak{r}}$ if and only if any right $R$-homomorphism from an ideal in $\mathscr{E}$ to $M$ may be extended to a right $R$-homomorphism from $R$ to $M$.

Proof. The necessity of this condition is, of course, obvious. Let us assume the condition holds and let $f: C \rightarrow M$ be an $R$-homomorphism, where $C$ is a right ideal of $R$ containing some ideal $A \in \mathscr{E}$. There exists a right $R$-homomorphism $g: R \rightarrow M$ extending the restriction $f_{A}$ of $f$ to $A$. But since $M$ is $\mathfrak{r}$-separated and $A \leqq{ }_{\mathrm{r}} C, f$ must coincide with $g$ on $C$.

Definition 2. We will say that an ideal $A$ of $R$ is weakly left invertible in the extension ring $R^{\prime}$ of $R$ if there exists a left $R$-submodule $B$ of $R^{\prime}$ such that $R \subseteq A B$ and $B A \subseteq R$. We call $B$ a weak left inverse of $A$ in $R^{\prime}$ and note that it is a twosided $R$-submodule of $R^{\prime}$, for $B R \subseteq B A B \subseteq R B \subseteq B$.

THEOREM 4. The following conditions on an ideal $A$ of $R$ are equivalent.

(1) $A$ is weakly left invertible in some extension ring of $R$.

(2) As a right $R$-module, $A$ is projective and finitely generated and $A \leqq R(R)$.

(3) $A \leqq R(R)$ and if $R^{\prime}$ is an extension ring of $R$ in which every right $R$ homomorphism from $A$ to $R$ is extendable to a right $R$-homomorphism from $R$ to $R^{\prime}$, then $A$ is weakly left invertible in $R^{\prime}$.

(4) $A$ is weakly left invertible in $R_{1}$.

Proof. Assume first of all that $A$ has a weak left inverse $B$ in some extension ring $R^{\prime}$ of $R$. Then there exist elements $a_{1}, \cdots, a_{n} \in A$ and $b_{1}, \cdots, b_{n} \in B$ such that

$$
a_{1} b_{1}+\cdots+a_{n} b_{n}=1
$$

and for each $i, \phi_{i}: a \rightarrow b_{i} a$ is a right $R$-homomorphism from $A$ to $R$. For each $a \in A$,

$$
a=1 a=a_{1} b_{1} a+\cdots+a_{n} b_{n} a=a_{1} \phi_{1}(a)+\cdots+a_{n} \phi_{n}(a)
$$

so that by Proposition 3.1 of Chapter VII of [4], as a right $R$-module, $A$ is projective and generated by $a_{1}, \cdots, a_{n}$. Let $\phi$ be a partial right $R$-homomorphism from $R$ to $R$ such that $A \subseteq \operatorname{ker} \phi$. If $x \in \operatorname{dom} \phi, \phi(x) A \subseteq \phi(x A) \subseteq \phi(A)=0$ so that $\phi(x) R \subseteq \phi(x) A B=0$ and therefore, $\phi(x)=\phi(x) 1=0$. Therefore, $A \leqq R(R)$.

Now assume that (2) holds and let $R^{\prime}$ be an extension ring of $R$ such that every right $R$-homomorphism from $A$ to $R$ is extendable to a right $R$-homomorphism from $R$ to $R^{\prime}$. By the proof of Proposition 3.1 of Chapter VII of [4], there exists a family of elements $\left\{a_{i}\right\}_{i=1}^{n}$ of $A$ and a family of right $R$-homomorphisms $\left\{\phi_{i}: A \rightarrow R\right\}_{i=1}^{n}$ such that for each $a \in A$

$$
a=\sum_{i=1}^{n} a_{i} \phi_{i}(a) \text {. }
$$

Each $\phi_{i}$ may be extended to $\psi_{i}: R \rightarrow R^{\prime}$. Set $b_{i}=\psi_{i}(1)$. Then for each $a \in A$, $b_{i} a=\psi_{i}(1) a=\phi_{i}(a)$ and 


$$
\left(1-\sum_{i=1}^{n} a_{i} b_{i}\right) a=0
$$

Since $A \leqq R(R)$, we conclude that $1=\sum_{i=1}^{n} a_{i} b_{i}$. Therefore, the left $R$-submodule of $R^{\prime}$ generated by $\left\{b_{i}\right\}_{i=1}^{n}$ is a weak left inverse of $A$ in $R^{\prime}$.

That (3) implies (4) and (4) implies (1) is immediate.

This theorem permits one to speak simply of the weakly left invertible ideals of $R$.

COROLlaRY. The set $\mathscr{E}_{2}$ of all weakly left invertible ideals of $R$ is closed under multiplication.

Proof. If $A$ and $C$ are in $\mathscr{E}_{2}$, then they have weak left inverses $B$ and $D$, respectively, in $R_{1}$ and $D B$ is a weak left inverse of $A C$ in $R_{1}$.

We will denote the torsion radical and the ring of quotients determined by $\mathscr{E}_{2}$ by $\mathfrak{r}_{2}$ and $R_{2}$, respectively.

LEMMA 5. Let $\mathscr{E}$ be a multiplicatively closed set of weakly left invertible ideals of $R$ defining the torsion radical $\mathfrak{x}$. If $\psi: R \rightarrow R^{\prime}$ is a ring homomorphism and if for every $A \in \mathscr{E}$, the ideal $\psi(A)$ of $\psi(R)$ is weakly left invertible in $R^{\prime}$, then $R^{\prime}$, turned into a right $R$-module in the natural way is $\mathfrak{r}$-separated.

Proof. If $u A=0$, where $u \in R^{\prime}$ and $A \in \mathscr{E}$, then if $B$ is a weak left inverse of $\psi(A)$ in $R^{\prime}$,

$$
u=u 1=u \psi(1) \in u(\psi(A) B)=(u A) B=0 .
$$

LEMMA 6. If the ideal $A$ of $R$ has a weak left inverse in the extension ring $R^{\prime}$ of $R$ then every right $R$-homomorphism $f: A \rightarrow R^{\prime}$ may be extended to a right $R$-homomorphism from $R$ to $R^{\prime}$.

Proof. There exist elements $a_{1}, \cdots, a_{n} \in A$ and $b_{1}, \cdots, b_{n} \in R^{\prime}$ such that

(1) $1=a_{1} b_{1}+\cdots+a_{n} b_{n}$,

(2) $a \rightarrow b_{i} a$ is a right $R$-homomorphism from $A$ to $R$.

Set $v=f\left(a_{1}\right) b_{1}+\cdots+f\left(a_{n}\right) b_{n} \in R^{\prime}$. Then, for any $a \in A$,

$$
\begin{aligned}
f(a) & =f(1 a)=f\left(a_{1} b_{1} a+\cdots+a_{n} b_{n} a\right) \\
& =f\left(a_{1}\right) b_{1} a+\cdots+f\left(a_{n}\right) b_{n} a \\
& =\left(f\left(a_{1}\right) b_{1}+\cdots+f\left(a_{n}\right) b_{n}\right) a=v a
\end{aligned}
$$

so that $f$ is the restriction of $\phi_{v}$ to $A$.

THEOREM 5. Let $\mathscr{E}$ be a multiplicatively closed set of weakly left invertible ideals of $R$, let $\mathfrak{r}$ denote the torsion radical of $\mathscr{C}_{R}$ determined by $\mathscr{E}$ and let $\kappa: 1_{\mathscr{R} R} \rightarrow S$ be a regular description of $\mathfrak{V}_{\mathfrak{r}}$. Then $\kappa_{R}$ is a monomorphism so that $S(R)$ may be considered as a true extension of $R$, every ideal in $\mathscr{E}$ has a weak left 
inverse in $S(R)$ and if $R^{\prime}$ is an extension ring of $R$ in which each ideal in $\mathscr{E}$ has a weak left inverse, then there exists a unique ring homomorphism $\chi: S(R) \rightarrow R^{\prime}$ extending $1_{R}$ and $\chi$ is a monomorphism.

Proof. By Theorem 4, each $A \in \mathscr{E}$ has the property $A \leqq R(R)$ so that $R$ is $\mathfrak{r}$ separated and therefore $S(R)$ may be considered as a true extension of $R$. That every ideal in $\mathscr{E}$ has a weak left inverse in $S(R)$ also follows from Theorem 4 . Now if $R^{\prime}$ is an extension ring of $R$ in which every ideal in $\mathscr{E}$ has a weak left inverse, then by Lemmas 5 and $6, R^{\prime} \in \mathfrak{Q}_{\mathfrak{r}}$ so that the existence and uniqueness of $\chi$ follow from Theorem 3 of $\S 5$. That $\chi$ is a monomorphism follows from the fact that $S(R)$ is an essential extension of $R$.

Under the conditions of Theorem 5, if $M$ is a module in $\mathscr{C}_{S(R)}$ such that $\mathfrak{r}(M)=M$, then $M=0$, for if $x \in M$, there exists an ideal $A \in \mathscr{E}$ such that $x A=0$ and if $B$ is a weak left inverse of $A$ in $S(R)$, then $x R \subseteq x A B=0$. Thus, by Theorem 2 of $\S 5$, $\mathrm{S}$ is equivalent to $\mathscr{C}_{R} \otimes_{R} S(R)$ and every $S(R)$-module, considered as an $R$-module, is in $Q_{\mathfrak{r}}$. Also, if $\rho$ is the canonical correspondence between the $R$-submodules of a module $M$ in $\mathscr{C}_{R}$ and the $S(R)$-submodules of $S(M)$ defined at the end of $\S 5$, and if $N \subseteq M$, then $\rho(N)=\kappa_{M}(N) \cdot S(R)$. Furthermore, we have the following proposition.

Proposition 5. Under the hypotheses of Theorem 5, if $M$ is a module in $\mathscr{C}_{R}$ and if $N^{\prime}$ is an $S(R)$-submodule of $S(M)$, then $N^{\prime}=\rho\left(\kappa_{M}^{-1}\left(N^{\prime}\right)\right)$.

Proof. If $x \in N^{\prime}$, since $\kappa_{M}(M) \leqq_{\mathfrak{r}} S(M)$, there exists $A \in \mathscr{E}$ such that $x A \subseteq \kappa_{M}(M)$. If $B$ is a weak left inverse of $A$ in $S(R)$, there exist elements $a_{1}, \cdots, a_{n} \in A$ and $b_{1}, \cdots, b_{n} \in B$ such that

$$
1=a_{1} b_{1}+\cdots+a_{n} b_{u}
$$

so that $x=x \cdot 1=\left(x a_{1}\right) b_{1}+\cdots+\left(x a_{n}\right) b_{n}$. Since each

$$
x a_{i} \in N^{\prime} \cap \kappa_{M}(M)=\kappa_{M}\left(\kappa_{M}^{-1}\left(N^{\prime}\right)\right), x \in \kappa_{M}\left(\kappa_{M}^{-1}\left(N^{\prime}\right)\right) \cdot S(R)=\rho\left(\kappa_{M}^{-1}\left(N^{\prime}\right)\right) .
$$

Therefore $N^{\prime}=\kappa_{M}\left(\kappa_{M}^{-1}\left(N^{\prime}\right)\right) \cdot S(R)=\rho\left(\kappa_{M}^{-1}\left(N^{\prime}\right)\right)$.

Definition 3. An ideal $A$ of $R$ will be said to be left invertible in an extension ring $R^{\prime}$ of $R$ if $A$ has a weak left inverse $B$ in $R^{\prime}$ with the extra property $B A \supseteq R$ so that $B A=R$. We then call $B$ a left inverse of $A$ in $R^{\prime}$.

Proposition 6. If $A$ is a weakly left invertible ideal of $R$, then the following conditions on $A$ are equivalent:

(1) $A$ is left invertible in some extension ring of $R$.

(2) For every module $M$ in $\mathscr{C}_{R}, M=\Sigma f(A)$, the sum being taken over all $f \in \operatorname{Hom}_{R}(A, M)$.

(3) $R=\Sigma f(A)$, the sum being taken over all $f \in \operatorname{Hom}_{R}(A, R)$.

(4) $A$ is left invertible in any extension ring of $R$ in which it is weakly left invertible. 
Proof. Assume first of all that $A$ has a left inverse $B$ in some extension ring $R^{\prime}$ of $R$. Then there exist elements $a_{1}, \cdots, a_{n} \in A$ and $b_{1}, \cdots, b_{n} \in B$ such that

$$
1=b_{1} a_{1}+\cdots+b_{n} a_{n} \text {. }
$$

If for each $i, \phi_{i}$ denotes the right $R$-homomorphism from $A$ to $R$ mapping each $a \in A$ onto $b_{i} a$, then for any $u \in M$,

$$
u=u 1=u b_{1} a_{1}+\cdots+u b_{n} a_{l:}=\phi_{u} \phi_{1}\left(a_{1}\right)+\cdots+\phi_{u} \phi_{n}\left(a_{n}\right) \in \sum_{f \in \operatorname{Hom}_{R^{(A, M)}}} f(A) .
$$

Now assume that (3) holds and that $A$ is weakly left invertible in an extension ring $R^{\prime}$ of $R$. Then

$$
1=f_{1}\left(a_{1}\right)+\cdots+f_{n}\left(a_{n}\right)
$$

where each $a_{i} \in A$ and each $f_{i} \in \operatorname{Hom}_{R}(A, R)$.

By Lemma 6 , for each $i \in I$, there exists $g_{i}: R \rightarrow R^{\prime}$ extending $f_{i}$. If we set $b_{i}=g_{i}(1)$, then for all $a \in A, f_{i}(a)=b_{i} a$ so that

$$
1=b_{1} a_{1}+\cdots+b_{n} a_{n} .
$$

But the set $B$ of all $b \in R^{\prime}$ such that $b A \subseteq R$ is the largest weak left inverse of $A$ in $R^{\prime}$, and we have just shown that $R \subseteq B A$ so that $B$ is a left inverse of $A$ in $R^{\prime}$.

This proposition permits one to speak simply of the left invertible ideals of $R$.

Corollary. The set $\mathscr{E}_{3}$ of all left invertible ideals of $R$ is closed under multiplication.

Proof. If $A, C \in \mathscr{E}_{3}$, then $A$ and $C$ have left inverses $B$ and $D$, respectively, in $R_{2}$. Then $D B$ is a left inverse of $A C$ in $R_{2}$.

We will denote the torsion radical and ring of quotients of $R$ determined by $\mathscr{E}_{3}$ by $\mathfrak{r}_{3}$ and $R_{3}$, respectively. Obviously, by Theorem 5 and Proposition $6, R_{3}$ is a true extension of $R$ contained in $R_{2}$ and every ideal in $\mathscr{E}_{3}$ is left invertible in $R_{3}$.

DEFINITION 4. If $\mathscr{E}$ is a set of ideals of $R$, then we will say that a module $M$ in $\mathscr{C}_{R}$ is $\mathscr{E}$-divisible if $M=M A$ for all $A \in \mathscr{E}$.

Proposition 7. If $A$ is a left invertible ideal of $R$ and if $M$ is a module in $\mathscr{C}_{R}$ such that every $R$-homomorphism from $A$ to $M$ is extendable to an $R$-homomorphism from $R$ to $M$, then $M=M A$.

Proof. If $u \in M$, by Proposition 6, there exist maps $f_{1}, \cdots, f_{n} \in \operatorname{Hom}_{R}(A, M)$ such that

$$
u=f_{1}\left(a_{1}\right)+\cdots+f_{n}\left(a_{n}\right)
$$

where the $a_{i}$ are in $A$. Then, for each $i$, there exists an $R$-homorphism $g_{i}: R \rightarrow M$ extending $f_{i}$ so that

$$
u=g_{1}\left(a_{1}\right)+\cdots+g_{n}\left(a_{n}\right)=g_{1}(1) a_{1}+\cdots+g_{n}(1) a_{n} \in M A .
$$


Corollary. If $\mathscr{E}$ is a multiplicatively closed set of left invertible ideals of $R$, defining the torsion radical $\mathfrak{r}$, then every module in $\tilde{\mathfrak{Q}}_{\mathfrak{r}}$ is $\mathscr{E}$-divisible.

DEFINITION 5. We will say that an ideal $A$ of $R$ is invertible in an extension ring $R^{\prime}$ of $R$ if $A$ has a left inverse $B$ in $R^{\prime}$ such that $A B \subseteq R$ so that $A B=B A=R$. We call $B$ an inverse of $A$ in $R^{\prime}$.

The set $\mathscr{E}_{4}$ of all ideals of $R$ that are invertible in some extension rings of $R$ is the intersection of $\mathscr{E}_{3}$ with the set of all right invertible ideals of $R$ so that it is closed under multiplication. We will denote the torsion radical and the ring of quotients of $R$ determined by $\mathscr{E}_{4}$ by $\mathfrak{r}_{4}$ and $R_{4}$, respectively. It is obvious that as an extension of $R, R_{4}$ may be embedded in $R_{3}$ and that every ideal in $\mathscr{E}_{4}$ is left invertible in $R_{4}$.

Proposition 8. If $A$ is an ideal of $R$ which is invertible in some extension ring $R^{\prime}$ of $R$ and if $M$ is a module in $\mathscr{C}_{R}$ such that $M=M A$, then every $R$ homomorphism from $A$ to $M$ is extendable to an $R$-homomorphism from $R$ to $M$.

Proof. There exist elements $a_{1}, \cdots, a_{n} \in A$ and $b_{1}, \cdots, b_{n}$ in the inverse $B$ of $A$ in $R^{\prime}$ such that

$$
1=a_{1} b_{1}+\cdots+a_{n} b_{n}
$$

Since $M=M A$, for each $i$,

$$
f\left(a_{i}\right)=u_{i 1} \lambda_{i 1}+\cdots+u_{i n} \lambda_{i n}
$$

where the $u_{i j} \in M$ and the $\lambda_{i j} \in A$. Since $B$ is an inverse of $A$ in $R^{\prime}$, each $\lambda_{i j} b_{i} \in R$. Set

$$
v=u_{11}\left(\lambda_{11} b_{1}\right)+\cdots+u_{1 n}\left(\lambda_{1 n} b_{1}\right)+\cdots+u_{n 1}\left(\lambda_{n 1} b_{n}\right)+\cdots+u_{n n}\left(\lambda_{n n} b_{n}\right) \in M .
$$

Then, for any $a \in A$,

$$
\begin{aligned}
f(a) & =f(1 a)=f\left(a_{1} b_{1} a+\cdots+a_{n} b_{n} a\right) \\
& =f\left(a_{1}\right)\left(b_{1} a\right)+\cdots+f\left(a_{n}\right)\left(b_{n} a\right) \\
& =\left(u_{11} \lambda_{11}+\cdots+u_{1 n} \lambda_{1 n}\right)\left(b_{1} a\right)+\cdots+\left(u_{n 1} \lambda_{n 1}+\cdots+u_{n n} \lambda_{n n}\right)\left(b_{n} a\right) \\
& =\left[u_{11}\left(\lambda_{11} b_{1}\right)+\cdots+u_{1 n}\left(\lambda_{1 n} b_{1}\right)+\cdots+u_{n 1}\left(\lambda_{n 1} b_{n}\right)+\cdots+u_{n n}\left(\lambda_{n n} b_{n}\right)\right] a \\
& =v a
\end{aligned}
$$

so that $f$ is the restriction of $\phi_{v}$ to $A$.

COROLLARY. If $\mathscr{E}$ is a multiplicatively closed set of ideals of $R$ that are invertible in some extension rings of $R$, defining the torsion radical $\mathfrak{r}$, then $\mathfrak{Q}_{\mathfrak{r}}$ is just the set of all $\mathfrak{r}$-separated and $\mathscr{E}$-divisible modules in $\mathscr{C}_{R}$. 
Proof. That every module in $\mathfrak{Q}_{\mathfrak{r}}$ is $\mathscr{E}$-divisible follows from the corollary of Proposition 7. Conversely, if $M$ is an $\mathfrak{r}$-separated and $\mathscr{E}$-divisible module, then $M \in \mathfrak{Q}_{\mathfrak{r}}$ by the preceding proposition and Lemma 4 .

THEOREM 6. If an ideal $A$ of $R$ is invertible and if $B$ is a left inverse of $A$ in the extension ring $R^{\prime}$ of $R$, then $B$ is an inverse of $A$ in $R^{\prime}$.

Proof. Since $B$ is a left inverse of $A$ in $R^{\prime}$,

$$
R^{\prime}=R R^{\prime} \subseteq A B R^{\prime} \subseteq A R^{\prime}
$$

so that $R^{\prime}=A R^{\prime}$.

By Proposition 8, applied to the mirror image of $R$, every left $R$-homomorphism from $A$ to $R^{\prime}$ is extendable to a left $R$-homomorphism from $R$ to $R^{\prime}$. Since $A$ is invertible, by Theorem 4 applied to the mirror image of $R$, as a left $R$-module, $A \leqq R(R)$. Then by this same theorem, $A$ has a weak right inverse $B^{\prime}$ in $R^{\prime}$. By Proposition 6, applied to the mirror image of $R$, there exists a right inverse $B^{\prime \prime}$ of $A$ in $R^{\prime}$. Then,

$$
B^{\prime \prime}=R B^{\prime \prime}=B A B^{\prime \prime}=B R=B .
$$

THEOREM 7. Let $\mathscr{E}$ be a set of invertible ideals of $R$ which is closed under multiplication and let $\mathfrak{r}$ and $\bar{R}$ denote the torsion radical and ring of quotients of $R$, respectively, determined by $\mathscr{E}$. Then the canonical homomorphism from $R$ to $\bar{R}$ is a monomorphism so that $\bar{R}$ may be considered as a true extension of $R$, every ideal in $\mathscr{E}$ is invertible in $\bar{R}$, and if $\psi: R \rightarrow R^{\prime}$ is a ring homomorphism such that for every $A \in \mathscr{E}$ the ideal $\psi(A)$ of $\psi(R)$ is invertible in $R^{\prime}$, then there exists a unique ring homomorphism from $R$ to $R^{\prime}$ extending $\psi$.

Proof. That the canonical homomorphism from $R$ to $\bar{R}$ is a monomorphism and that every ideal in $\mathscr{E}$ is invertible in $\bar{R}$ follow from Theorem 5, Proposition 6 and Theorem 6. By Lemma $5, R^{\prime}$, turned into a right $R$-module in the natural way, is $\mathfrak{r}$-separated. If $A \in \mathscr{E}$ and if $B$ is the inverse of $\psi(A)$ in $R^{\prime}$, then

$$
R^{\prime}=R^{\prime} \psi(R)=R^{\prime} B \psi(A) \subseteq R^{\prime} \psi(A)=R^{\prime} A
$$

so that $R^{\prime}=R^{\prime} A$. By the corollary of Proposition $8, R^{\prime} \in \mathfrak{Q}_{\mathfrak{r}}$. Then, by Theorem 3 of $\S 5$, there exists a unique ring homomorphism from $\bar{R}$ to $R^{\prime}$ extending $\psi$.

COROLlaRY. The right ring of quotients of $R$ and the left ring of quotients of $R$ determined by $\mathscr{E}$ are equivalent ring extensions of $R$.

We notice of course that if $R$ is a commutative ring, the notions of weakly left invertible ideal and invertible ideal coincide.

7. Pure submodules. Let $N$ be an ideal of $R$. Every module in $\mathscr{C}_{R / N}$ may be turned into a right $R$-module in a natural way and then every $R / N$-homomorphism is also an $R$-homomorphism. This defines a functor $T$ from $\mathscr{C}_{R / N}$ to $\mathscr{C}_{R}$. 
As was stated in $\S 3, T$ has a regular left adjoint $S$ defined by a natural transformation $\kappa: 1_{\mathscr{C}_{R}} \rightarrow T S$ and for each $A$ in $\mathscr{C}_{R}, \kappa_{A}$ may be identified with the natural homomorphism of $A$ onto $A / A N$. The exact structure of $\mathscr{C}_{R / N}$ then induces an injective structure $\mathfrak{B}_{N}\left(\mathfrak{S}_{N}, \mathfrak{Q}_{N}\right)$, where $\mathfrak{S}_{N}$ is the class of all those homomorphisms $f: A \rightarrow B$ in $\mathscr{C}_{R}$ which induce a monomorphism from $A / A N$ to $B / B N$ and where the class $\mathfrak{Q}^{\prime}{ }_{N}$ of all modules $C$ in $\mathscr{C}_{R}$ such that $C N=0$ and $C$, considered as an $R / N$-module, is an injective module of $\mathscr{C}_{R / N}$, is a basis of injectives of $\mathfrak{B}_{N}$.

If $A \in \mathfrak{Q}_{N}$, then there exist $R$-modules $C$ and $B$ such that $C \in \mathfrak{Q}^{\prime}$ and $C=A \oplus B$. Since $C N=0$, we have $A N=B N=0$ so that $A$ may be considered as an $R / N$ module and as such, it is a direct summand of $C$ and therefore also an ordinary injective module of $\mathscr{C}_{R / N}$. Therefore, $\mathfrak{Q}_{N}=\mathfrak{Q}_{N}^{\prime}$.

It is obvious that if we denote the radical of $\mathscr{C}_{R}$ determined by $\mathfrak{P}_{N}$ by $\mathfrak{r}_{N}$, then for any module $A$ in $\mathscr{C}_{R}, \mathfrak{r}_{N}(A)=A N$. Then, $\mathfrak{S}_{N}$ consists of those homomorphisms $f: A \rightarrow B$, where $\operatorname{ker} f \subseteq A N$ and where the natural injection of $f(A)$ into $B$ is in $\mathfrak{S}_{N}$. If $A \subseteq B$, then the kernel of the natural homomorphism $\psi$ of $A / A N$ into $B / B N$ is $(B N \cap A) / A N$ so that $\psi$ is a monomorphism if and only if $B N \cap A=A N$, i.e., the natural injection of $A$ into $B$ is in $\Im_{N}$, which we denote by $A \gamma_{N} B$, if and only if $B N \cap A=A N$. Thus, a homomorphism $f: A \rightarrow B$ is in $\mathfrak{S}_{N}$ if and only if ker $f \subseteq A N$ and $f(A) \gamma_{N} B$.

Now let $\mathscr{E}$ be any set of ideals of $R$ and set

$$
\mathfrak{B}_{8}\left(\mathfrak{S}_{\&}, \mathfrak{Q}_{8}\right)=\sup _{N \in \mathscr{8}} \mathfrak{B}_{N}
$$

The radical of $\mathfrak{B}_{\mathscr{E}}$, which we denote by $\mathfrak{r}_{\mathscr{E}}$, is the intersection of all the $\mathfrak{r}_{N}, N \in \mathscr{E}$. Furthermore, by Proposition 5, $\S 1, \bigcup_{N \in \delta} \mathfrak{Q}_{N}$ is a subbasis of injectives of $\mathfrak{B}_{\S}$ and

$$
\mathfrak{\Xi}_{8}=\bigcap_{N \in \delta} \mathfrak{S}_{N}
$$

so that the homomorphisms in $\mathfrak{S}_{\mathcal{S}}$, which we call the $\mathscr{E}$-pure homomorphisms of $\mathscr{C}_{R}$, are those homomorphisms $f: A \rightarrow B$ for which $\operatorname{ker} f \subseteq \mathfrak{r}_{8}(A)$ and $B N \cap A=A N$ for all $N \in \mathscr{E}$. We will refer to this last condition by saying that $f(A)$ is $\mathscr{E}$-pure in $B$ and by writing $f(A) \gamma_{\varepsilon} B$. Notice that for any module $A$ in $\mathscr{C}_{R}, \hat{\mathfrak{r}}_{\mathscr{E}}(A)$ is the largest $\mathscr{E}$-divisible submodule of $A$. The definitions and arguments given in $\S 2$ of [10] extend almost word for word to yield a theory of $\gamma_{8}$-essential extensions of modules.

By Theorem 1 of $\S 3$, there is a coarsest regular injective structure $\mathfrak{B}_{\delta}^{*}\left(\Im_{\delta}^{*}, \mathfrak{Q}_{\delta}^{*}\right)$ finer than $\mathfrak{V}_{\mathscr{E}}, \mathfrak{V}_{\mathscr{\delta}}^{*}$ determines the radical $\mathfrak{r}_{\mathscr{E}}$, and $\mathfrak{S}_{\mathscr{E}}^{*}$ contains the class of all homomorphisms $f: A \rightarrow B$ in $\mathfrak{S}_{\delta}$ for which $\mathfrak{r}_{\delta}(B / f(A))=B / f(A)$. We notice that if $f: A \rightarrow B$ is such that $\operatorname{ker} f \subseteq \mathfrak{r}_{\delta}(A)$ and $\mathfrak{r}_{\delta}(B / f(A))=B / f(A)$, then $f$ is not necessarily in $\mathfrak{\Im}_{\delta}^{*}$ : one must also have that $f(A) \gamma_{\delta} B$ for $f$ to be in $\Im_{\varepsilon}^{*}$.

It would be interesting to know general conditions under which $\mathfrak{B}_{\&}=\mathfrak{B}_{\&}^{*}$, for example, in the following particular cases: 
(1) $\mathscr{E}$ is the set of all maximal ideals of $R\left(\mathfrak{r}_{\mathscr{\delta}}(R)\right.$ is then the Brown and McCoy radical of $R$ ).

(2) $\mathscr{E}$ is the set of annihilators of simple right $R$-modules $\left(\mathfrak{r}_{\mathscr{\delta}}(R)\right.$ is then the Jacobson-Chevalley radical of $R$ ).

(3) $\mathscr{E}$ is the set of all prime ideals of $R\left(\mathfrak{r}_{\mathscr{E}}(R)\right.$ is then the lower nil radical of $\left.R\right)$.

(4) $\mathscr{E}$ is the set of all finite products of ideals in one of the preceding sets.

We note that when $\mathscr{E}$ is a multiplicatively closed set of non-null ideals of a principal ideal ring $R$, then it was shown in [10] that $\mathfrak{B}_{\&}^{*}=\mathfrak{B}_{\mathcal{\&}}$.

We now assume that $\mathscr{E}$ has the following property:

H. If $N_{1}, N_{2} \in \mathscr{E}$, then there exists $N \in \mathscr{E}$ such that $N \subseteq N_{1} \cap N_{\tilde{2}}$. Then, for any module $A$ in $\mathscr{C}_{R},\{A N\}_{N \in \mathscr{E}}$ is a basis of the filter of neighborhoods of 0 of a topology $\mathscr{T}(A, \mathscr{E})$ of $A$ compatible with addition and scalar multiplication. This topology has the following properties:

(i) $\mathfrak{r}_{\mathscr{E}}(A)$ is the closure of 0 in $(A, \mathscr{T}(A, \mathscr{E}))$.

(ii) If $A \subseteq B$ and if $\phi$ denotes the natural homomorphism of $B$ onto $B / A$, then the closure of $A$ in $(B, \mathscr{T}(B, \mathscr{E}))$ is

$$
\bigcap_{N \in \mathscr{E}}(A+B N)=\phi^{-1}\left(\mathfrak{r}_{\mathscr{E}}(B / A)\right) \text {. }
$$

(iii) If $A \gamma_{\mathscr{E}} B$, then $\mathscr{T}(B, \mathscr{E})$ induces $\mathscr{T}(A, \mathscr{E})$ on $A$.

(iv) $\mathscr{T}(A, \mathscr{E})$ is discrete if and only if $A N=0$ for some $N \in \mathscr{E}$.

LEMMA 1. If $\mathscr{T}(A, \mathscr{E})$ is Hausdorff and if $(B, \mathscr{T})$ is a completion of $(A, \mathscr{T}(A, \mathscr{E}))$ then $A \gamma_{\mathscr{E}} B$.

Proof. We notice first of all that for each $N \in \mathscr{E}$, the closure $\overline{A N}$ of $A N$ in $(B, \mathscr{T})$ is an $R$-submodule of $B$ and that $\{\overline{A N}\}_{N \in \mathscr{B}}$ is a basis of the filter of neighborhoods of 0 in $(B, \mathscr{T})$. Suppose that $a \in B N \cap A$, where $N \in \mathscr{E}$, so that $a=b_{1} n_{1}+\cdots b_{r} n_{r}$ where $b_{i} \in B$ and $n_{i} \in N$. There exists a neighborhood $V$ of 0 in $(B, \mathscr{T})$ such that $V \cap A \subseteq A N$, and by the remark made above, we may assume that $V$ is an $R$-submodule of $B$. Since $A$ is dense in $(B, \mathscr{T})$, for each $i=1,2, \cdots, r$, there exists $a_{i} \in\left(b_{i}+V\right) \cap A$. Set $a_{i}=b_{\imath}+c_{i}$, where $c_{\imath} \in V$. Then

$$
a=\left(a_{1} n_{1}+\cdots+a_{r} n_{r}\right)-\left(c_{1} n_{1}+\cdots+c_{r} n_{r}\right)
$$

so that $a-\left(a_{1} n_{1}+\cdots+a_{r} n_{r}\right) \in A \cap V \subseteq A N$ and therefore $a \in A N$.

Now if $A$ is any module in $\mathscr{C}_{R}$, we denote by $\phi_{A}$ the natural homomorphism of $A$ onto $A / \mathfrak{r}_{\mathscr{\delta}}(A)=A^{\prime}$. Since $\mathfrak{r}_{\mathscr{E}}\left(A^{\prime}\right)=0, \mathscr{T}\left(A^{\prime}, \mathscr{E}\right)$ is Hausdorff and we may speak of the completion $\left(S_{\mathscr{E}}(A), \mathscr{T}_{A}\right)$ of the topological $R$-module $\left(A^{\prime}, \mathscr{T}\left(A^{\prime}, \mathscr{E}\right)\right)$. Let $\kappa_{A}$ denote the product of $\phi_{A}$ by the natural injection of $A^{\prime}$ into $S_{\mathscr{E}}(A)$. If $f: A \rightarrow B$, $f$ induces a unique $g: A^{\prime} \rightarrow B^{\prime}$ and $g$ is obviously a continuous $R$-homomorphism from $\left(A^{\prime}, \mathscr{T}\left(A^{\prime}, \mathscr{E}\right)\right)$ to $\left(B^{\prime}, \mathscr{T}\left(B^{\prime}, \mathscr{E}\right)\right)$ so that $g$ may be extended in a unique fashion to a continuous $R$-homomorphism $S_{\mathscr{\delta}}(f)$ from $\left(S_{\mathscr{E}}(A), \mathscr{T}_{A}\right)$ to $\left(S_{\varepsilon}(B), \mathscr{T}_{B}\right)$. We notice that $S_{\delta}(f) \kappa_{A}=\kappa_{B} f$. It is then obvious that $S_{\mathcal{E}}$ is a functor from $\mathscr{C}_{R}$ into itself and that $\kappa$ is a natural transformation from $1_{\mathscr{C}_{R}}$ to $S$ 
THEOREM 1. $\left(S_{\mathscr{\delta}}, \kappa\right)$ is a functorial description of an injective structure $\mathfrak{V}_{\mathscr{E}}^{\#}\left(\mathscr{S}_{\mathscr{E}}^{\#}, \mathfrak{Q}_{\mathscr{E}}^{\#}\right)$ which is finer than $\mathfrak{B}_{\mathscr{E}}$. The class $\mathfrak{Q}_{\mathscr{E}}^{\prime}$ of all modules $Q$ in $\mathscr{C}_{R}$ on which there exists a Hausdorff topology which is compatible with addition and scalar multiplication and which is complete and coarser than $\mathscr{T}(Q, \mathscr{E})$ is a basis of injectives of $\mathfrak{B}_{\mathscr{E}}^{\#}$.

Proof. For any module $A$ in $\mathscr{C}_{R}$, by Lemma $1, A^{\prime} \gamma_{\mathscr{E}} S_{\mathscr{E}}(A)$ so that $\mathscr{T}_{A}$ and $\mathscr{T}\left(S_{\mathscr{E}}(A), \mathscr{E}\right)$ both induce $\mathscr{T}\left(A^{\prime}, \mathscr{E}\right)$ on $A^{\prime}$ and therefore by the corollary of Proposition 9, Chapter II, of [3], $\mathscr{T}_{A}$ is coarser than $\mathscr{T}\left(S_{\mathscr{\delta}}(A), \mathscr{E}\right)$. Thus we see that $S_{\mathscr{E}}(A) \in \mathfrak{Q}_{\mathscr{\delta}}^{\prime}$. Now let $f$ be an $R$-homomorphism from $A$ to $Q \in \mathfrak{Q}_{\mathscr{\delta}}^{\prime}$. There exists a Hausdorff topology $\mathscr{T}$ on $Q$ which is compatible with addition and scalar multiplication and which is complete and coarser than $\mathscr{T}(Q, \mathscr{E})$. It is then obvious that $\mathfrak{r}_{\mathscr{E}}(Q)=0$ so that there exists a unique $R$-homomorphism $g: A^{\prime} \rightarrow Q$ such that $f=g \phi_{A}$. It is obvious that $g$ is a continuous $R$-homomorphism from $\left(A^{\prime}, \mathscr{T}\left(A^{\prime}, \mathscr{E}\right)\right)$ to $(Q, \mathscr{T}(Q, \mathscr{E}))$ so that, a fortiori, it is a continuous $R$-homomorphism from $\left(A^{\prime}, \mathscr{T}\left(A^{\prime}, \mathscr{E}\right)\right)$ to $(Q, \mathscr{T})$. Since $(Q, \mathscr{T})$ is Hausdorff and complete, $g$ may be extended uniquely to a continuous $R$-homomorphism $h$ from $\left(S_{\mathscr{E}}(A), \mathscr{T}_{A}\right)$ to $(Q, \mathscr{T})$ (but $h$ may not be the only $R$-homomorphism from $S_{\mathscr{E}}(A)$ to $Q$ extending $g$ so that $\mathfrak{B}_{\mathscr{E}}^{\#}$ may not be regular) so that $f=h \kappa_{A}$. Then, by Proposition 2 of $\S 1, Q_{\mathscr{E}}^{\prime}$ is a basis of injectives of an injective structure of $\mathfrak{V}_{\mathscr{E}}^{\#}$ of $\mathscr{C}_{R}$ which is described by $\left(\kappa, S_{\mathscr{E}}\right)$. If $A$ is any module in $\mathscr{C}_{R}$, there exists $\phi: A \rightarrow Q$ in $\mathfrak{S}_{\mathscr{E}}$, where $Q \in \mathfrak{Q}_{\mathscr{E}}$ and since, by Lemma $1, \kappa_{A} \in \mathfrak{S}_{\mathscr{E}}$, there exists $f: S_{\mathscr{E}}(A) \rightarrow Q$ such that $\phi=f \kappa_{A}$. Therefore, $\mathfrak{B}_{\mathscr{E}}^{\#}$ is finer than $\mathfrak{B}_{\mathscr{E}}$, by Proposition 5 of $\S 2$.

THEOREM 2. $\mathfrak{B}_{\mathscr{\delta}}^{\#}$ is coarser than $\mathfrak{B}_{\mathcal{E}}^{*}$ and the following three statements are equivalent:

(1) $\mathfrak{B}_{\delta}^{\#}=\mathfrak{B}_{\mathscr{\delta}}^{*}$.

(2) For any $\mathfrak{r}_{\mathscr{E}}$-separated module $A$ in $\mathscr{C}_{R}, \mathscr{T}\left(S_{\mathscr{E}}(A), \mathscr{E}\right)=\mathscr{T}_{A}$.

(3) $\mathfrak{Q}_{\mathscr{E}}^{*}$ is precisely the class of all $\mathfrak{r}_{\mathscr{E}}$-separated modules $A$ in $\mathscr{C}_{R}$ such that $(A, \mathscr{T}(A, \mathscr{E}))$ is complete.

Proof. If $A$ is any $\mathfrak{r}_{\mathscr{B}}$-separated module in $\mathscr{C}_{R}$, then there exists an extension $A^{\prime \prime}$ of $A$ such that $A^{\prime \prime} \in \mathfrak{Q}_{\mathscr{E}}^{*}, A \gamma_{\mathscr{E}} A^{\prime \prime}$ and $\mathfrak{r}_{\mathscr{E}}\left(A^{\prime \prime} \mid A\right)=A^{\prime \prime} \mid A$. Then $(A, \mathscr{T}(A, \mathscr{E}))$ is a dense topological $R$-submodule of $\left(A^{\prime \prime}, \mathscr{T}\left(A^{\prime \prime}, \mathscr{E}\right)\right)$ so $1_{A}$ may be extended to a continuous $R$-monomorphism $f$ from $\left(A^{\prime \prime}, \mathscr{T}\left(A^{\prime \prime}, \mathscr{E}\right)\right.$ ) to $\left(S_{\mathscr{E}}(A), \mathscr{T}_{A}\right)$ (which we consider as a true extension of $A$ ). Since $f$ is obviously the only $R$-homomorphism from $A^{\prime \prime}$ to $S_{\mathscr{E}}(A)$ extending $1_{A}$, from here on, we consider $f$ as a natural injection of $A^{\prime \prime}$ into $S_{\mathscr{E}}(A)$. It is obvious then that $A^{\prime \prime}$ is just the inverse image of $\mathfrak{r}_{\mathscr{\varepsilon}}\left(S_{\mathscr{\delta}}(A) / A\right)$ by the natural homomorphism of $S_{\delta}(A)$ onto $S_{\delta}(A) / A$. By Proposition 5 of $\S 2$, we conclude that $\mathfrak{B}_{\mathscr{E}}^{*}$ is finer than $\mathfrak{B}_{\mathscr{E}}^{\#}$. Now $\mathfrak{B}_{\mathscr{\delta}}^{*}=\mathfrak{B}_{\mathscr{E}}^{\#}$ means that for any $\mathfrak{r}_{\mathscr{E}}$-separated module $A, A^{\prime \prime}=S_{\mathscr{E}}(A)$. Then $1_{A^{\prime \prime}}$ is a continuous homomorphism from $\left(A^{\prime \prime}, \mathscr{T}\left(A^{\prime \prime}, \mathscr{E}\right)\right)$ to $\left(S_{\mathscr{E}}(A), \mathscr{T}_{A}\right)$ so that it may be extended uniquely to a continuous homomorphism $g$ from $\left(S_{\mathscr{\delta}}\left(A^{\prime \prime}\right), \mathscr{T}_{A^{\prime \prime}}\right)$ to $\left(S_{\mathscr{\delta}}(A), \mathscr{T}_{A}\right)$ (here again we 
consider $S_{\delta}\left(A^{\prime \prime}\right)$ as a true extension of $\left.A^{\prime \prime}\right)$. But $\left(S_{\mathscr{\delta}}\left(A^{\prime \prime}\right), \mathscr{T}_{A^{\prime \prime}}\right)$ is a completion of the topological $R$-module $(A, \mathscr{T}(A, \mathscr{E}))$ so that $g$ must be a bicontinuous isomorphism and therefore $\mathscr{T}\left(S_{\mathscr{E}}(A), \mathscr{E}\right)=\mathscr{T}_{A}$. Now assume that (2) holds and let $A \in Q_{\mathscr{\delta}}^{*}$. Then $\mathscr{T}_{A}=\mathscr{T}\left(S_{\mathscr{\delta}}(A), \mathscr{E}\right)$ so that $\mathrm{r}_{\mathscr{E}}\left(S_{\mathscr{E}}(A) / A\right)=S_{\mathscr{\delta}}(A) / A$ and therefore the natural injection of $A$ into $S_{\mathscr{\delta}}(A)$ is in $\mathfrak{S}_{\mathscr{\delta}}^{*}$. Then $A$ is a direct summand of $S_{\mathscr{\delta}}(A)$, i.e., $S_{\mathscr{\delta}}(A)=A \oplus C$, and since $C \cong S_{\mathscr{E}}(A) / A, \mathfrak{r}_{\mathscr{\delta}}(C)=C$, contradicting the fact that $S_{\mathscr{E}}(A)$ is $\mathfrak{r}_{\mathscr{E}}$-separated, unless $C=0$ and $A=S_{\mathscr{\delta}}(A)$, so that $\mathscr{T}(A, \mathscr{E})$ is complete. That every $\mathfrak{r}_{\mathscr{E}}$-separated module $A$ for which $\mathscr{T}(A, \mathscr{E})$ is complete is in $\mathfrak{Q}_{\varepsilon}^{*}$ is obvious. Finally, if (3) holds, for any $\mathfrak{r}_{\xi}$-separated module $A,\left(A^{\prime \prime}, \mathscr{T}\left(A^{\prime \prime}, \mathscr{E}\right)\right)$ is a completion of $(A, \mathscr{T}(A, \mathscr{E}))$ so that $\left(A^{\prime \prime}, \mathscr{T}\left(A^{\prime \prime}, \mathscr{E}\right)\right)=\left(S_{\delta}(A), \mathscr{T}_{A}\right)$ and therefore $\mathfrak{B}_{\mathscr{E}}^{*}=\mathfrak{B}_{\mathscr{E}}^{\#}$.

\section{REFERENCES}

1. R. Baer, Abelian groups that are direct summands of every containing abelian group, Bull. Amer. Math. Soc. 46 (1940), 800-806.

2. N. Bourbaki, Algèbre, Eléments de mathématique, Chapitre 8, Actualités Sci. Indust. no. 1261, Hermann, Paris, 1958.

3. - Topologie générale, éléments de mathématique, Chapitres I, II, Actualités Sci. Indust. no. 858-1152, Hermann, Paris, 1951.

4. H. Cartan and S. Eilenberg, Homological algebra, Princeton Univ. Press, Princeton, N.J., 1956.

5. G. Findlay and J. Lambek, A generalized ring of quotients. I, II, Canad. Math. Bull. 1 (1958), 77-85, 155-167.

6. R. Godement, Théorie des faisceaux, Actualités Sci. Indust. no. 1252, Hermann, Paris, 1958.

7. A. Heller, Homological algebra in abelian categories, Ann. of Math. (2) 68 (1958), 484-525.

8. R. E. Johnson, The extended centralizer of a ring over a module, Proc. Amer. Math. Soc. 2 (1951), 891-895.

9. D. M. Kan, Adjoint functors, Trans. Amer. Math. Soc. 87 (1958), 294-329.

10. J.-M. Maranda, On pure subgroups of abelian groups, Arch. Math. 11 (1960), 1-13.

11. Y. Utumi, On quotient rings, Osaka Math. J. 8 (1956), 1-18.

UNIVERSITE DE MONTREAL, Montreal, Canada 